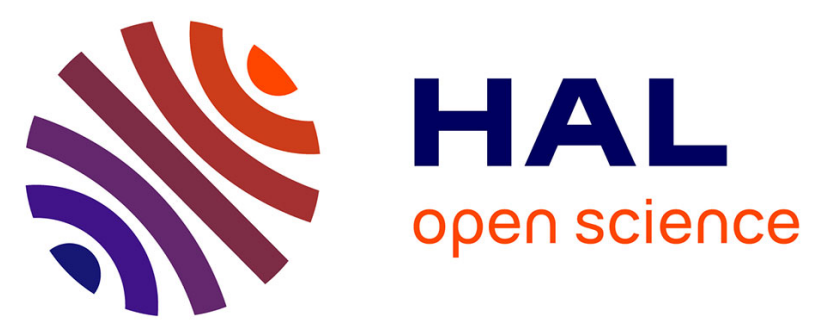

\title{
A comparison between Monte Carlo simulation and finite-volume scheme for reliability assessment of multi-state physics systems
}

\author{
Yan-Hui Lin, Yan-Fu Li, Enrico Zio
}

\section{- To cite this version:}

Yan-Hui Lin, Yan-Fu Li, Enrico Zio. A comparison between Monte Carlo simulation and finite-volume scheme for reliability assessment of multi-state physics systems. Reliability Engineering and System Safety, 2018, 174, pp.1 - 11. 10.1016/j.ress.2018.01.008 . hal-01786573

\section{HAL Id: hal-01786573}

https://hal-centralesupelec.archives-ouvertes.fr/hal-01786573

Submitted on 27 Apr 2020

HAL is a multi-disciplinary open access archive for the deposit and dissemination of scientific research documents, whether they are published or not. The documents may come from teaching and research institutions in France or abroad, or from public or private research centers.
L'archive ouverte pluridisciplinaire HAL, est destinée au dépôt et à la diffusion de documents scientifiques de niveau recherche, publiés ou non, émanant des établissements d'enseignement et de recherche français ou étrangers, des laboratoires publics ou privés. 


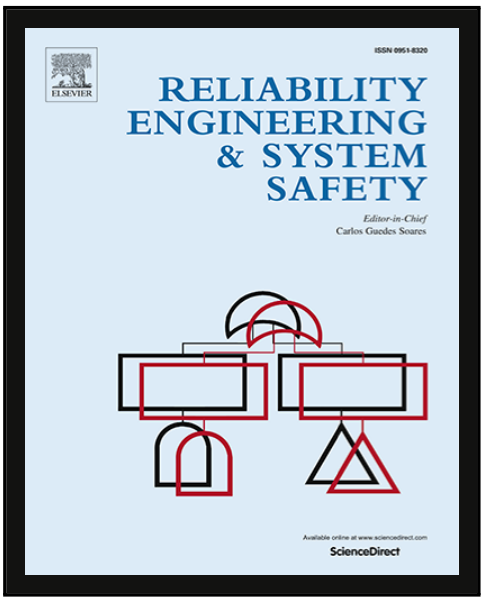

A Comparison between Monte Carlo Simulation and Finite-Volume Scheme for Reliability Assessment of Multi-State Physics Systems

Yan-Hui Lin , Yan-Fu Li , Enrico Zio

PII: S0951-8320(18)30036-X

DOI: 10.1016/j.ress.2018.01.008

Reference: RESS 6049

To appear in: Reliability Engineering and System Safety

Received date:

14 April 2016

Revised date:

17 January 2018

Accepted date:

19 January 2018

Please cite this article as: Yan-Hui Lin , Yan-Fu Li , Enrico Zio , A Comparison between Monte Carlo Simulation and Finite-Volume Scheme for Reliability Assessment of Multi-State Physics Systems, Reliability Engineering and System Safety (2018), doi: 10.1016/j.ress.2018.01.008

This is a PDF file of an unedited manuscript that has been accepted for publication. As a service to our customers we are providing this early version of the manuscript. The manuscript will undergo copyediting, typesetting, and review of the resulting proof before it is published in its final form. Please note that during the production process errors may be discovered which could affect the content, and all legal disclaimers that apply to the journal pertain. 


\section{highlights}

- Dependencies among degradation processes in MSPS are modeled by PDMP.

- A comparative study of MC simulation and FV scheme for reliability assessment of MSPS is conducted.

- Two case studies regarding a real industrial system are considered.

- Guidelines for the selection of the two approaches are provided.

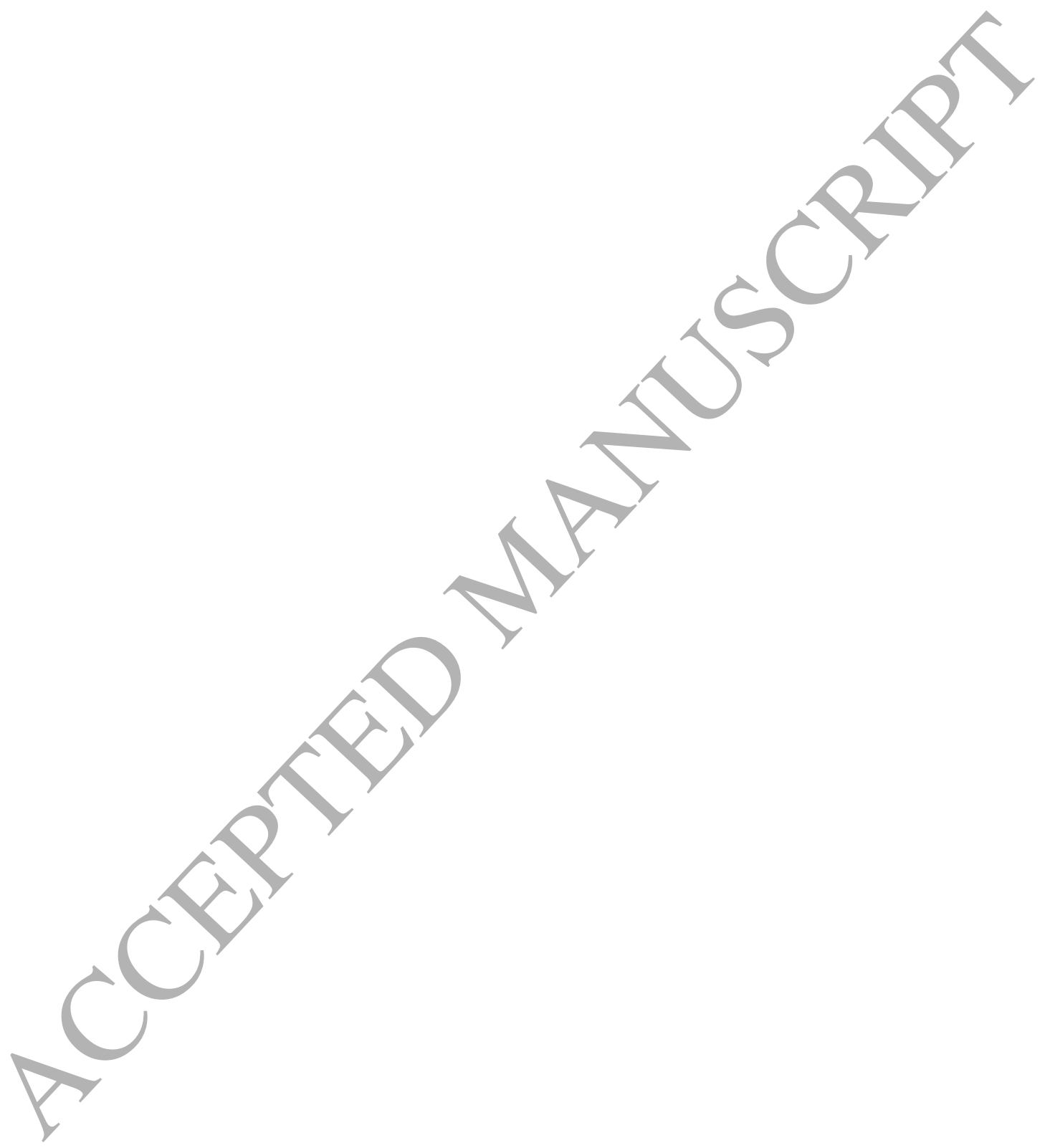




\title{
A Comparison between Monte Carlo Simulation and
}

\section{Finite-Volume Scheme for Reliability Assessment of Multi-State}

\author{
Physics Systems \\ Yan-Hui Lin ${ }^{1}$, Yan-Fu Li ${ }^{2, *}$ and Enrico $\mathrm{Zio}^{3,4}$ \\ ${ }^{1}$ School of Reliability and Systems Engineering, Beihang University, Beijing, China \\ ${ }^{2}$ Department of Industrial Engineering, Tsinghua University, Beijing, China \\ ${ }^{3}$ Chair on Systems Science and the Energetic Challenge, Foundation EDF, at CentraleSupelec, \\ France \\ ${ }^{4}$ Politecnico di Milano, Italy \\ Email address: liyanfu@tsinghua.edu.cn
}

\begin{abstract}
Multi-state physics systems (MSPS) modeling framework incorporates multi-state models that describes the systems/degradation/maintenance process through transitions among discrete states, and physics-based models that describe the degradation process within the states by using physics knowledge and equations. In previous works, piecewise-deterministic Markoy process (PDMP) has been adopted to treat the system dynamics and the degradation dependence in MSPS. For reliability assessment, Monte Carlo simulation and finite-volume method are two widely used numerical approaches to solve PDMP. In the present work, a comparative study considering different evaluation criteria of the two approaches is conducted on two representative case studies. We provide clear guidelines for the selection of the two approaches.
\end{abstract}

Keywords: multi-state model, physics-based model, dependent degradation processes, piecewise-deterministic Markov process, Monte Carlo simulation method, finite-volume scheme.

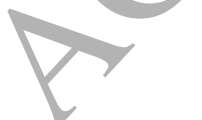

PBMs

MSMs

PDMP

$\mathrm{MC}$

FV

RHRS

$\mathrm{KB}$

\section{Acronyms}

Physics-based models

Multi-state models

Piecewise-deterministic Markov process

Monte Carlo

Finite-volume

Residual heat removal system

Kilobytes 


\section{Notations}

$\boldsymbol{L} \quad$ Group of degradation processes modeled by PBMs

$\boldsymbol{K} \quad$ Group of degradation processes modeled by MSMs

$\overrightarrow{X_{L_{m}}}(t) \quad$ Time-dependent continuous variables of degradation process $L_{m}$

$\overrightarrow{f_{L_{m}}} \quad$ Physics equations

$\theta_{L_{m}} \quad$ Parameters in $\overrightarrow{f_{L_{m}}}$

$\underset{{L_{m}}_{m}}{\longrightarrow} \quad$ Degradation threshold

$Y_{K_{n}}(t) \quad$ State variable of degradation process $K_{n}$

$\lambda_{i} \quad$ Transition rate of $Y_{K_{n}}(t)$

$\theta_{K_{n}} \quad$ Parameters in $\lambda_{i}$

$S_{K_{n}} \quad$ Finite state set of degradation process $K_{n}$

$\vec{Z}(t) \quad$ Degradation state of the system

$\boldsymbol{\theta}_{\boldsymbol{K}} \quad$ Parameters used in $\boldsymbol{K}$

$\lambda_{\vec{\imath}}\left(\vec{\jmath} \mid \vec{X}(t), \boldsymbol{\theta}_{\boldsymbol{K}}\right)$ Transition rate from state $\vec{\imath}$ to $\vec{\jmath}$

$\boldsymbol{\theta}_{\boldsymbol{L}} \quad$ Parameters used in $\boldsymbol{L}$

$\overrightarrow{f_{L} \vec{Y}(t)}\left(\vec{X}(t), t \mid \boldsymbol{\theta}_{\boldsymbol{L}}\right)$ Deterministic physics equations in $\boldsymbol{L}$

\section{INTRODUCTION}

Most systems degrade over time and experience intermediate degradation states before complete failure. For some highly reliable components/systems (e.g. pumps and valves in nuclear power plants), their degradation and/or failure data are often limited. In this situation, multi-state physics systems (MSPS) modeling framework [1] which incorporates multi-state models (MSMs) [2-7] and physics-based models (PBMs) [8-11] can be applied. A MSM describes the degradation process in a discrete way, supported by material science knowledge [1], degradation and/or failure data [3] from historical field collection or degradation tests. Giorgio et al. [3] modeled the degradation process by a Markov model in which the transition probabilities between unit states depend on both the current age and the current degradation level. Chryssaphinou et al. [2] employed discrete-time semi-Markov chains to model component degradation processes and used the vector of paired processes of the 
semi-Markov chain and the corresponding backward recurrence time process to describe the behavior of the whole system. Li and Pham [4] developed a generalized multi-state degraded system reliability model subject to multiple competing failure processes, including two degradation processes, and random shocks. Moghaddass and Zuo [7] employed nonhomogeneous continuous-time semi-Markov processes to model degradation and developed supervised parametric and nonparametric estimation methods to estimate the maximum likelihood estimators of the main characteristics of the model. Please refer to [5] for other studies on MSMs. A PBM aims at developing an integrated mechanistic description of the component/system life consistent with the underlying real degradation mechanisms (e.g. wear, corrosion, cracking, etc.) by using physics knowledge and equations [12]. Daigle and Goebel [8] developed PBMs for the degradation processes of the pneumatic valves based on mass and energy balances and the underlying damage mechanisms. Daigle and Goebel [9] employed PBMs to model impeller wear and bearing wear of centrifugal pumps. Keedy and Feng [10] modeled fatigue crack growth of the stents due to cyclic stresses by using the mechanics-based approach. Reggiani et al. [11] built a PBM for hot-carrier stress degradation based on the linear drain current.

In reality, systems are often subject to multiple degradation processes. These degradation processes can be dependent under certain circumstances, e.g. when the degradation dynamics of some components depend on the degradation state of other components [13], or the various degradation processes share the same influencing factors [14]. This renders the system reliability analysis and prediction a challenging problem. Peng et al. [15] considered two dependent failure processes modeled as stochastic processes. Wang and Pham [16] applied time-varying copulas for describing the dependence between the degradation processes modeled by statistical distributions. Yang et al. [17] modeled the components dependence through the joint distribution of failure time. Straub [18] used a dynamic Bayesian network to represent the dependence between degradation processes modeled by multi-state models. The dependence is handled in different ways according to the types of degradation models involved. However, none of the previous methods can treat the degradation dependency in a system whose degradation processes are modeled by PBMs and MSMs.

Piecewise-deterministic Markov process (PDMP) can be employed to integrate PBMs and MSMs for dealing with the degradation dependence among different components, as shown in our previous preliminary study [19]. The PDMP, firstly introduced by Davis in [20, 21], and further studied by Jacobsen [22] and Cocozza-Thivent [23] is a family of Markov processes involving deterministic evolution punctuated by random jumps. It is a general model and includes many other models (e.g. semi-Markov process, Markov process, etc.) as special cases. Marseguerra and Zio [24] applied PDMP approach for dynamic reliability assessment of a heated hold-up tank system, whereas Chiquet et al. [25] used PDMP to model fatigue crack in a structural component. Zhang et al. [26] applied PDMP to an offshore oil production system and demonstrated its high modeling ability. However, due to the complex behavior of PDMP, analytical solutions are difficult to obtain [24]. 
The Monte Carlo (MC) simulation method and finite-volume (FV) approach are two widely used approaches for solving PDMP models to evaluate reliability quantities. Zhang et al. [27] have used the MC simulation method to assess the safety and production availability of an offshore oil production system. An FV scheme is a feasible alternative for solving PDMP by discretizing its continuous variable space and the time space. It can achieve the results comparable to the MC simulation method, but in significantly shorter computing times in certain cases. Lair et al. [28] have developed a FV scheme to optimize the preventive maintenance of air-conditioning systems used in trains. Cocozza-Thivent et al. [29] have proposed an explicit FV scheme for dynamic reliability assessment. An implicit FV scheme has been proposed by Eymard et al. [30] to assess the marginal distribution of a process describing the time evolution of a hybrid system. Lin et al. [31] have extended a FV scheme to quantify the fuzzy system reliability considering epistemic uncertainty and degradation dependency for low dimensional problems with simple equations describing the deterministic evolution of PBMs. Eymard et al. [32] applied FV scheme for sensitivity analysis in dynamic reliability models. Based upon previous published works, we summarize the characteristics of both evaluation methods as follows: MC simulation method is readily to be implemented, however, requires considerable amount of computing time; FV scheme can be more efficient in low dimension problems, but is relatively difficult to implement and deploy. To the knowledge of the authors, there is no clear conclusion about when to utilize which method. Therefore, a systematic comparative study considering different evaluation criteria is necessary to provide useful guidelines for the researchers and the practitioners.

The major difference between present work and the previous works $[19,33]$ is that this is a systematic comparative study on the methods for reliability assessment of multi-state physics systems (MSPSs) considering different evaluation criteria. This type of study is useful for the following research works as well as for practice purpose. To the knowledge of the authors, it has not been done in the literature and there is no clear conclusion about which method should be applied when encountering different system types (e.g. large/small sized, with complex/simple degradation equations). The methodological contribution of this work is mainly on the aspect of method selection: we provide clear guidelines for choosing the two methods, considering several evaluation criteria, i.e. accuracy, computation time, memory consumption, efficiency, scope of application and ease of implementation.

The reminder of this article is organized as follows. Section 2 introduces the PDMP modeling for MSPS reliability assessment. The procedures of MC simulation method and FV scheme to solve the model are presented in Section 3. Section 4 presents the evaluation criteria, the case study and the comparison of the two methods. Section 5 concludes the work.

\section{PDMP MODELING OF MSPS RELIABILITY ASSESSMENT}

\subsection{Assumptions of system and degradation models}


We consider a multi-component system. For modeling degradation processes, different types of degradation models can be included: PBMs, probabilistic \& statistical models, MSM models and continuous degradation models. In this work, we combine PBMs and MSMs under a general setting with no constraint on system topology (e.g. series, parallel, series-parallel, etc.). A component may have multiple degradation processes and each may be modeled by a PBM or a MSM. Therefore, a component may involve multiple PBMs and multiple MSMs. Note that the degradation processes in one component or different components are possibly dependent and the system topology does not affect the results shown later. We classify them into two groups: (1) $\boldsymbol{L}=\left\{L_{1}, L_{2}, \ldots, L_{M}\right\}$ modeled by $M$ PBMs; (2) $K=\left\{K_{1}\right.$, $\left.K_{2}, \ldots, K_{N}\right\}$ modeled by $N$ MSMs, where $L_{m}, m=1,2, \ldots, M$ and $K_{n}, n=$ $1,2, \ldots, N$ are the indexes of the degradation processes. We follow the definitions of PBMs and MSMs given in [33].

\subsubsection{PBMs [33]}

For $L_{m} \in \boldsymbol{L}$, its degradation level is denoted by vector $\overrightarrow{X_{L_{m}}}(t)$, which contains $d_{L_{m}}$ continuous variables involving degradation variables such as crack length [10] and wear area [9], and physical variables such as velocity and force [8]. Its evolution in time is characterized by a system of first-order differential equations $\overrightarrow{X_{L_{m}}}(t)=$ $\overrightarrow{f_{L_{m}}}\left(\overrightarrow{X_{L_{m}}}(t), t \mid \theta_{L_{m}}\right)$, i.e. physics equations, where $\theta_{L_{m}}$ reprsents influencing factors and the related coefficients. Note that higher-order differential equations can be converted into a system of large number of first-order differential equations by introducing extra variables. $L_{m}$ is regarded as failure as long as one degradation variable $x_{L_{m}}^{i}(t)$ in $\overrightarrow{X_{L_{m}}}(t)$ exceeds its predefined failure threshold $x_{L_{m}}^{i}$.

\subsubsection{MSMs [33]}

For $K_{n} \in \boldsymbol{K}$, its degradation level is denoted by $Y_{K_{n}}(t)$, taking values from a finite state set denoted by $S_{K_{n}}=\left\{0,1, \ldots, d_{K_{n}}\right\}$, where $d_{K_{n}}$ is the perfect functioning state and 0 is the complete failure state. Markov processes [3] are employed which are widely used in practice as MSMs. The transition rates $\lambda_{i}\left(j \mid \theta_{K_{n}}\right), \forall i, j \in S_{K_{n}}, i>$ $j$ characterize the transition probabilities from $i$ to state $j$ caused by degradation, where $\theta_{K_{n}}$ represents the influencing factors and the related coefficients. $K_{n}$ is considered as failure when $Y_{K_{n}}(t)$ reaches the state 0 . 
In our current model, there is no assumption about the dependence between $\theta_{K_{n}}$ and $\theta_{L_{m}}$, since they belongs to different degradation models. However, dependency can be considered. For example, if some influencing factors, such as temperature and humidity, are considered in both the PBM and the MSM, then, $\theta_{K_{n}}$ and $\theta_{L_{m}}$ contain same factors. Furthermore, $\lambda_{i}\left(j \mid \theta_{K_{n}}\right)$ can be a function of time, if the time factor belongs to $\theta_{K_{n}}$

\subsection{PDMP modeling [19]}

The dependence between degradation processes may exist within each group and between the two groups (e,g, the evolution of $\overrightarrow{X_{L_{m}}}(t)$ may be influenced by the degradation states of $\overrightarrow{X_{m^{\prime}}}(t), m \neq m^{\prime}$ and $Y_{K_{n}}(t)$; the transition rates of $Y_{K_{n}}(t)$ may be influenced by the degradation states of $Y_{K_{n^{\prime}}}(t), n \neq n^{\prime}$ and $\overrightarrow{X_{L_{m}}}(t)$ ). An illustration of a system with two dependent degradation processes is shown in Fig. 1, where the further degraded states of $K_{1}\left(L_{1}\right)$ lead to higher degradation rates of $L_{1}$ (higher transition rates of $K_{1}$ to step to further degraded states).

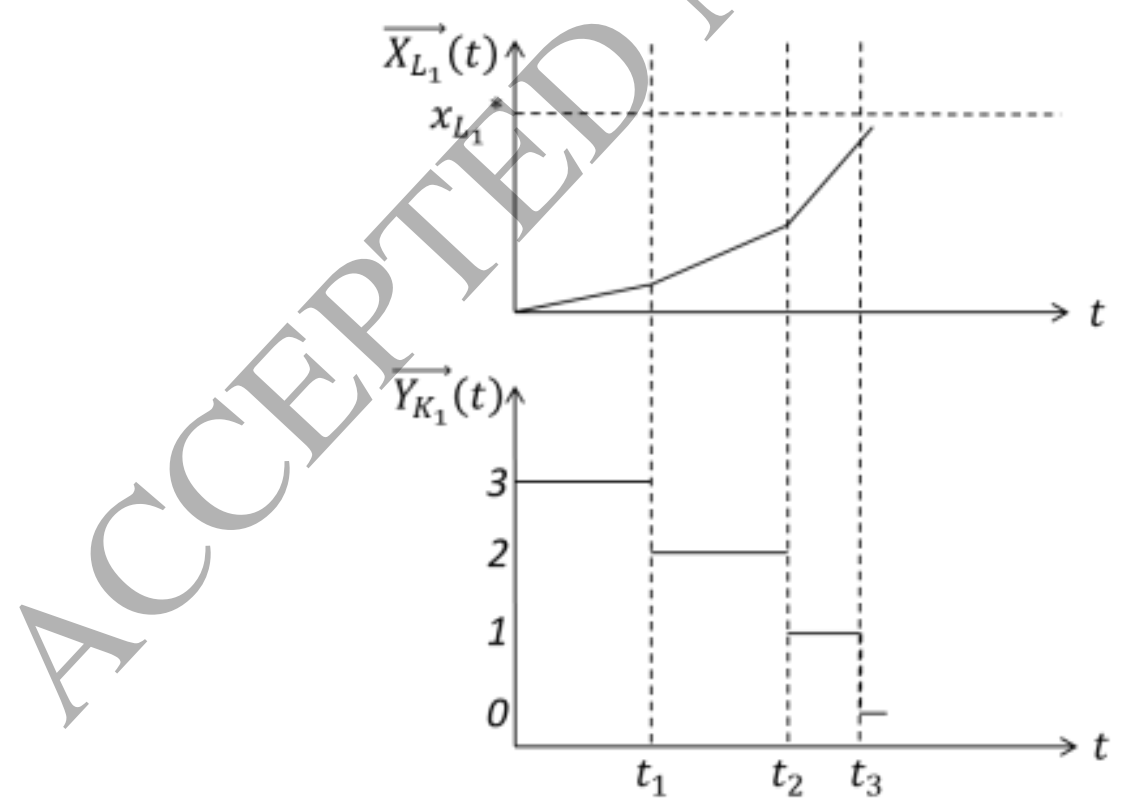

Fig. 1. An illustrative example of a system with two dependent degradation processes. Top Figure: degradation process of $L_{1}$; Bottom Figure: degradation process of $K_{1}$. 
In this particular case, the degradation rate of $\overrightarrow{X_{L_{1}}}(t)$ changes at the same time when $Y_{K_{1}}(t)$ changes. However, this does not necessarily happen in all cases since the degradation rate of $\overrightarrow{X_{L_{1}}}(t)$ may also depend on other influencing factors and the related coefficients in the physics equations.

PDMP can be employed to model this type of interdependence [19] as shown in eqs. (2) and (3). Let

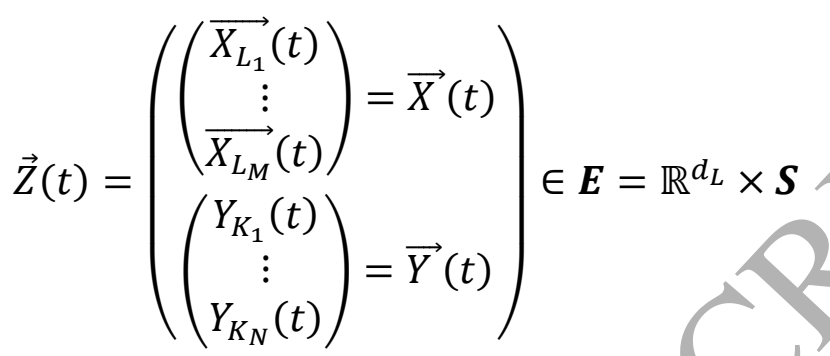

denote the overall degradation processes of the system where $\boldsymbol{E}$ is the space combining $\mathbb{R}^{d_{L}}\left(d_{L}=\sum_{m=1}^{M} d_{L_{m}}\right)$ and $\boldsymbol{S}$. The evolution of $\vec{Z}(t)$ involves two parts:

(1) the stochastic behavior of $\vec{Y}(t)$, which is governed by the transition rates depending on the degradation states of all the degradation processes in system as follows:

$$
\begin{gathered}
\lim _{\Delta t \rightarrow 0} P\left(\vec{Y}(t+\Delta t)=\vec{\jmath} \mid \vec{X}(t), \vec{Y}(t)=\vec{\imath}, \boldsymbol{\theta}_{\boldsymbol{K}}=\bigcup_{n=1}^{N} \boldsymbol{\theta}_{K_{n}}\right) / \Delta t \\
=\lambda_{\vec{\imath}}\left(\vec{\jmath} \mid \vec{X}(t), \boldsymbol{\theta}_{\boldsymbol{K}}\right), \forall t \geq 0, \vec{\imath}, \vec{\jmath} \in \boldsymbol{S}, \vec{\imath} \neq \vec{\jmath}
\end{gathered}
$$

(2) the deterministic behavior of $\vec{X}(t)$ between two consecutive jumps of $\vec{Y}(t)$, which is described by the deterministic physic equations depending on the degradation states of all the degradation processes in system as follows:

$$
\begin{aligned}
\vec{X}(t) & =\left(\begin{array}{c}
\overrightarrow{X_{L_{1}}}(t) \\
\vdots \\
\overrightarrow{X_{L_{M}}^{\cdot}}(t)
\end{array}\right)=\left(\begin{array}{c}
\overrightarrow{f_{L_{1}} \vec{Y}(t)}\left(\vec{X}(t), t \mid \boldsymbol{\theta}_{L_{1}}\right) \\
\vdots \\
\overrightarrow{f_{L_{M}} \vec{Y}(t)}\left(\vec{X}(t), t \mid \boldsymbol{\theta}_{L_{M}}\right)
\end{array}\right) \\
& =\overrightarrow{f_{L}^{\vec{Y}}(t)}\left(\vec{X}(t), t \mid \boldsymbol{\theta}_{\boldsymbol{L}}=\bigcup_{m=1}^{M} \boldsymbol{\theta}_{L_{m}}\right)
\end{aligned}
$$

Let $T_{k}$ denote the $k$-th transition time of the process $\vec{Y}(t) .\left\{\overrightarrow{Z_{k}}, T_{k}\right\}_{k \geq 0}$ is, then, a Markov renewal process [23] defined on the space $\boldsymbol{E} \times \mathbb{R}^{+}$, since the probability that the whole system will step to state $\vec{\jmath}$ from state $\vec{\imath}, \vec{\imath}, \vec{\jmath} \in \boldsymbol{E}, \vec{\imath} \neq \vec{\jmath}$ in the time interval $\left[T_{n}, T_{n}+\Delta t\right]$, given $\left\{\overrightarrow{Z_{k}}, T_{k}\right\}_{k \leq n}$ is:

$$
P\left[\overrightarrow{Z_{n+1}}=\vec{\jmath}, T_{n+1} \in\left[T_{n}, T_{n}+\Delta t\right] \mid\left\{\overrightarrow{Z_{k}}, T_{k}\right\}_{k \leq n-1},\left\{\overrightarrow{Z_{n}}=\vec{\imath}, T_{n}\right\}\right]
$$




$$
\begin{gathered}
=P\left[\overrightarrow{Z_{n+1}}=\vec{\jmath}, T_{n+1} \in\left[T_{n}, T_{n}+\Delta t\right] \mid \overrightarrow{Z_{n}}=\vec{\imath}\right] \\
\forall n \geq 0, \vec{\imath}, \vec{\jmath} \in \boldsymbol{E}, \vec{\imath} \neq \vec{\jmath}
\end{gathered}
$$

Let $\mathcal{F}$ denote the predefined space of the failure states of $\vec{Z}(t)$ : then, the system reliability at time $t$ is defined as follows:

$$
R(t)=P[\vec{Z}(s) \notin \mathcal{F}, \forall s \leq t]
$$

To consider a general setting, $\mathcal{F}$, is dependent on system topology which is problem-specific and can be determined by using reliability analysis tools such as fault tree analysis.

\section{METHODS FOR RELIABILITY ASSESSMENT}

\subsection{MC simulation method for solving PDMP}

Analytically solving the PDMP is a difficult task due to the complexity in the system behavior [24], with stochastic state transitions occurring in the components modeled by MSMs and time-dependent evolutions of the characteristic variables in the components modeled by PBMs. MC simulation method is widely used to solve PDMP in practice, which is readily to be implemented. Here, we employ the MC simulation method proposed in [33] for system reliability estimation. Appendix A contains a detailed description of the procedures of the MC simulation method.

\subsection{FV scheme for solving PDMP}

The MC simulation method is conceptually easy to apply and without particular restrictions on the dimension of PDMP. On the contrary, it can be quite time-consuming because of the repetition of many trials in order to get a satisfactory accuracy in the system reliability estimate.

An FV scheme discretizing the state space of the continuous variables and the time space of PDMP is an alternative that in certain cases can lead to results comparable to the MC simulation method, but in significantly shorter computing times. Here, we employ an explicit FV scheme for system reliability estimation [29]. Appendix B contains a detailed description of the FV method.

\section{Comparative Study}

\subsection{Evaluation criteria}

The evaluation criteria for the comparative study are accuracy, computation time, memory consumption, scope of application and ease of implementation. The first three attributes are quantitative and the rests are qualitative.

To compute accuracy, we use the results obtained by the MC simulation method with $10^{5}$ trials as reference values $x_{\text {reference }}$ and compute the relative change of 
the results $x$ obtained by another method (i.e. MC simulation method with different trials or FV scheme): Relative change $\left(x, x_{\text {reference }}\right)=\left(x-x_{\text {reference }}\right) /$ $x_{\text {reference }}$, where $x$ is the obtained system reliability.

The efficiency is also an important measure of performance. A method is more efficient if it can produce results comparable with the other, but with less computation time (here measured in seconds).

The memory consumption refers to the amount of digital information stored in the computer during the calculation and is measured in kilobytes (KB).

For the scope of application, we consider two case studies: one with high dimension and the other with low dimension, since the two methods mainly differ in their capacity of treating different dimensions of the problem.

The ease of implementation describes how easy it is to implement a method in practice.

\subsection{Numeric experiment design}

All the numerical experiments are carried out in MATLAB on a PC with an Intel Core 2 Duo CPU at $3.06 \mathrm{GHz}$ and a RAM of 3.07 GB.

We consider MC simulations with $10^{3}, 10^{4}$ and $10^{5}$ trials (for ease of reference, hereafter named MC1, MC2 and MC3, respectively). The parameters of the FV scheme are problem-dependent. Their tuning can be achieved by gradually decreasing the space step and the time step. To/compare the two methods, the parameter setting of FV scheme is first assigned such that it can lead to similar results as MC3, which gives the most accurate results that are used for reference. Then, we consider several parameter settings around it.

\subsection{Test cases and results}

We consider an important subsystem of a residual heat removal system of a nuclear power plant [37], consisting of a pneumatic valve and a centrifugal pump, which are used in conjunction in a variety of domains for fluid delivery $[8,38]$. The degradation model of the pump is the one originally considered in [19] while that of the valve is the physics-based model presented in [8]. Dependence is considered, as a result of discussions with experts: the degradation of the pump can lead it to vibrate [38], which will, in turn, cause the vibration of the valve and, therefore, aggravate the degradation process of the latter [39].

The degradation process of the centrifugal pump is modeled by a continuous-time homogeneous Markov chain with constant transition rates as shown in Fig. 2:

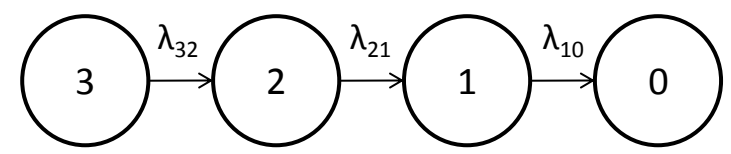

Fig. 2. Degradation process of the pump [19].

The perfect functioning state is denoted with the label ' 3 ' and ' 0 ' is the label of the 
complete failure state. The vibration of the pump caused by degradation is classified into two levels: 'smooth' and 'rough' [40], corresponding to the degradation states ' 2 ' and ' 1 ', respectively. Let $Y_{p}(t)$ denote the degradation state of the pump at time $t$ and $\boldsymbol{S}_{p}=\{0,1,2,3\}$ denote the degradation states set. The values of the degradation transition rates are presented in Table I.

Table I Values of the degradation transition rates of the pump

\begin{tabular}{|l|l|}
\hline Parameter & Value \\
\hline$\lambda_{32}$ & $6.00 \mathrm{e}-3 / \mathrm{s}$ \\
\hline$\lambda_{21}$ & $6.00 \mathrm{e}-3 / \mathrm{s}$ \\
\hline$\lambda_{10}$ & $6.00 \mathrm{e}-3 / \mathrm{s}$ \\
\hline
\end{tabular}

The pneumatic valve refers to a normally-closed and gas-actuated valve with a linear cylinder actuator, which has been studied in [8, 41] and [42] by physics-based modeling. A simplified scheme of the valve is shown in Fig. 3.

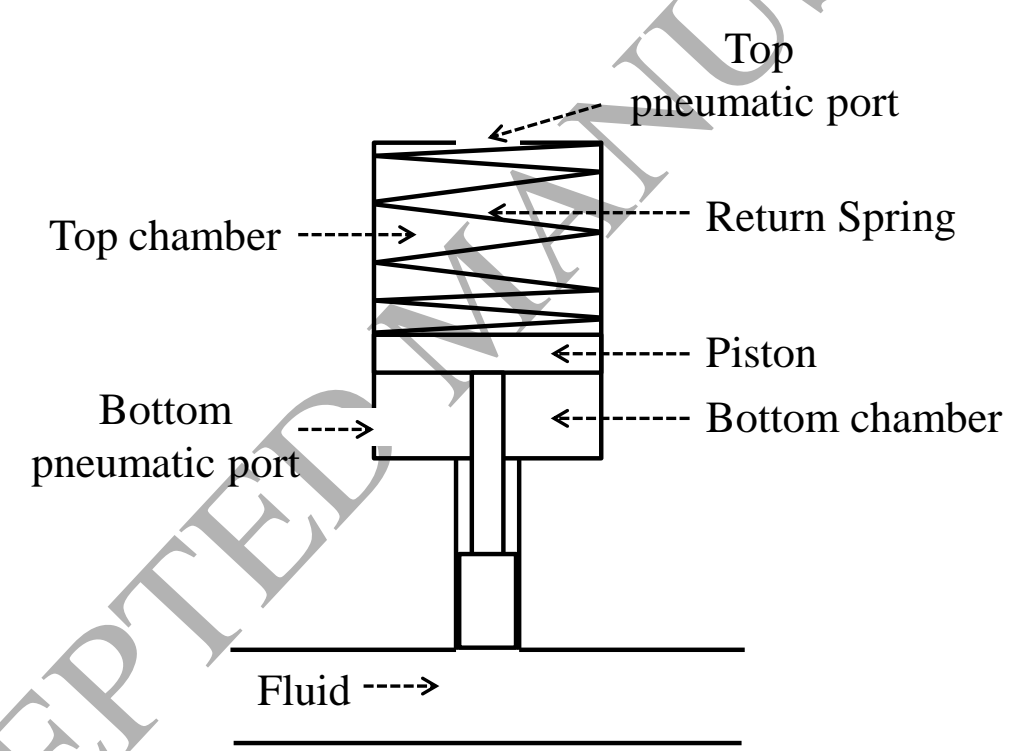

Fig. 3. Simplified scheme of the pneumatic valve [41].

Two case studies considering two different degradation mechanisms of the valve will be carried out in the following section.

\subsubsection{Case 1}

A common degradation mechanism of the valve is the internal leakage from the seal surrounding the piston [42]. Owing to this, the pneumatic gas can flow between the two chambers therefore influencing the response time and behavior of the valve. The degradation variable of the valve is the equivalent orifice area of the internal leakage of the piston, denoted by $L(t)$, and the degradation process of the valve at 
time $t$ is described by the following vector:

$$
\overrightarrow{X_{v}}(t)=\left(\begin{array}{c}
L(t) \\
x(t) \\
v(t) \\
m_{t}(t) \\
m_{b}(t) \\
t
\end{array}\right)
$$

where $x(t)$ is the position of the valve, $v(t)$ is the velocity of the valve, $m_{t}(t)$ is the mass of the gas in the top chamber, $m_{b}(t)$ is the mass of the gas in the bottom chamber and $t$ is the running time of the valve. The derivatives of these variables are represented by:

$$
\overrightarrow{\dot{X}_{v}}(t)=\left(\begin{array}{c}
\dot{L}(t) \\
v(t) \\
a(t) \\
f_{t}(t) \\
f_{b}(t) \\
1
\end{array}\right)
$$

where $a(t)$ is the valve acceleration, $f_{t}(t)$ and $f_{b}(t)$ are the mass flows going into the top and bottom chambers, respectively. The details of the physic functions governing the evolutions of the above variables are as follows:

$$
\dot{L}(t)=w r v(t)^{2}
$$

where $w$ is the wear coefficient,

$$
\begin{gathered}
a(t)=\frac{1}{m}\left[\left(p_{b}(t)-p_{t}(t)\right)\left(A_{p}-L(t)\right)-m g+\right. \\
\left.-k\left(x(t)+x_{0}\right)-r v(t)+F_{c}(x(t))\right]
\end{gathered}
$$

where

$$
p_{b}(t)=\frac{m_{b}(t) R_{g} T}{V_{b 0}+A_{p} x(t)}
$$

is the gas pressure on the bottom of the piston,

$$
p_{t}(t)=\frac{m_{t}(t) R_{g} T}{V_{t 0}+A_{p}\left(L_{s}-x(t)\right)}
$$

is the gas pressure on the top of the piston,

$$
F_{c}(x(t))= \begin{cases}k_{c}(-x(t)), & \text { if } x(t)<0 \\ 0, & \text { if } 0 \leq x(t) \leq L_{s} \\ -k_{c}\left(x(t)-L_{s}\right), & \text { if } x(t)>L_{s}\end{cases}
$$

is the contact force,

$$
\begin{array}{r}
f_{t}(t)=f_{g}\left(u_{t}(t), p_{t}(t), A_{s}\right)+f_{g}\left(p_{b}(t), p_{t}(t), L(t)\right) \\
f_{b}(t)=f_{g}\left(u_{b}(t), p_{b}(t), A_{s}\right)+f_{g}\left(p_{t}(t), p_{b}(t), L(t)\right)
\end{array}
$$

where $u_{t}(t)$ and $u_{b}(t)$ are the pressures on the top and bottom pneumatic ports, respectively, alternating between $P_{\text {sup }}$ and $P_{\text {atm }}$ depending on the command (opening command: $u_{t}(t)=P_{a t m}$ and $u_{b}(t)=P_{\text {sup }}$; closing command: $u_{t}(t)=$ $P_{\text {sup }}$ and $\left.u_{b}(t)=P_{\text {atm }}\right)$, and $f_{g}$ defines the gas flow through an orifice as follows: 


$$
f_{g}\left(p_{1}, p_{2}, A\right)= \begin{cases}\varepsilon P C_{S} A \sqrt{\frac{\gamma}{z R_{g} T}\left(\frac{2}{\gamma+1}\right)^{\frac{\gamma+1}{\gamma-1}}}, & \text { if } \delta \leq\left(\frac{2}{\gamma+1}\right)^{\frac{\gamma}{\gamma-1}} \\ \varepsilon P C_{S} A \sqrt{\frac{\gamma}{z R_{g} T}\left(\frac{2}{\gamma-1}\right)\left(\delta^{\frac{2}{\gamma}}-\delta^{\frac{\gamma+1}{\gamma}}\right),} & \text { if } \delta>\left(\frac{2}{\gamma+1}\right)^{\frac{\gamma}{\gamma-1}}\end{cases}
$$

where $\left\{\begin{array}{l}P=\max \left(p_{1}, p_{2}\right) \\ \delta=\frac{\min \left(p_{1}, p_{2}\right)}{\max \left(p_{1}, p_{2}\right)} \\ \varepsilon=\operatorname{sgn}\left(p_{1}-p_{2}\right)\end{array}\right.$.

The parameters definitions and numerical values related to the internal leakage degradation are presented in Table II below.

Table II Parameter Definitions and Values of Internal Leakage variables [8]

\begin{tabular}{|l|l|}
\hline Parameter - Definition & Value \\
\hline$g-$ acceleration due to gravity & $9.8 \mathrm{~m} / \mathrm{s}$ \\
\hline$P_{s u p}-$ supply pressure & $5.27 \mathrm{e} 6 \mathrm{~Pa}$ \\
\hline$P_{a t m}-$ atmospheric pressure & $1.01 \mathrm{e} 5 \mathrm{~Pa}$ \\
\hline$m-$ mass of the moving parts of the valve & $50 \mathrm{~kg}$ \\
\hline$r-$ coefficient of kinetic friction & $6.00 \mathrm{e} 3 \mathrm{Ns} / \mathrm{m}$ \\
\hline$k-$ spring constant & $4.80 \mathrm{e} 4 \mathrm{~N} / \mathrm{s}$ \\
\hline$k_{c}-$ large spring constant associated with the flexible seals & $1.00 \mathrm{e} 8 \mathrm{~N} / \mathrm{s}$ \\
\hline$x_{0}-$ amount of spring compression when the valve is closed & $0.254 \mathrm{~m}$ \\
\hline$L_{s}-$ fully open position of the valve & $0.1 \mathrm{~m}$ \\
\hline$A_{p}-$ surface area of the piston & $8.10 \mathrm{e}-3 \mathrm{~m}^{2}$ \\
\hline$V_{t 0}-$ minimum gas volume of the top chamber & $8.11 \mathrm{e}-4 \mathrm{~m}^{3}$ \\
\hline$V_{b 0}-$ minimum gas yolume of the bottom chamber & $8.11 \mathrm{e}-4 \mathrm{~m}^{3}$ \\
\hline$R_{g}-$ gas constant for the pneumatic gas & $296 \mathrm{~J} / \mathrm{K} / \mathrm{kg}$ \\
\hline$T-$ ideal gas temperature & $293 \mathrm{~K}$ \\
\hline$\gamma-$ ration of specific heats & 1.4 \\
\hline$z-$ gas compressibility factor & 1 \\
\hline$A_{S}-$ orifice area of the pneumatic port & $1.00 \mathrm{e}-5 \mathrm{~m}^{2}$ \\
\hline$W-$ wear coefficient & $6 \mathrm{e}-9 \mathrm{~m} / \mathrm{N}$ \\
\hline$C_{s}-$ flow coefficient & 0.1 \\
\hline
\end{tabular}

At the initial stage, the valve is set to the fully closed position with the values:

$$
\overrightarrow{X_{v}}(0)=\left(\begin{array}{c}
L(0) \\
0 \\
0 \\
\frac{P_{s u p}\left(L_{s} A_{p}+V_{t 0}\right)}{R_{g} T} \\
\frac{P_{a t m} V_{b 0}}{R_{g} T} \\
0
\end{array}\right)
$$

The threshold $L^{*}$ for the internal leakage of the piston $L(t)$ is defined as the value 
above which $\left(L(0)>L^{*}\right)$ the valve cannot reach the fully open position within the $15 \mathrm{~s}$ time limit after an opening command is executed at time $t=0 \mathrm{~s}$. The size of the internal leakage is assumed to be constant during the opening procedure $(\dot{L}(t)=0$, $0 \leq t \leq 15$ ) [42] to obtain a conservative threshold of $L^{*}=3.20 e-6 \mathrm{~m}^{2}$ in this case. The behavior of the valve within 15 s with different values of $L(0)$ is shown in Fig. 4.

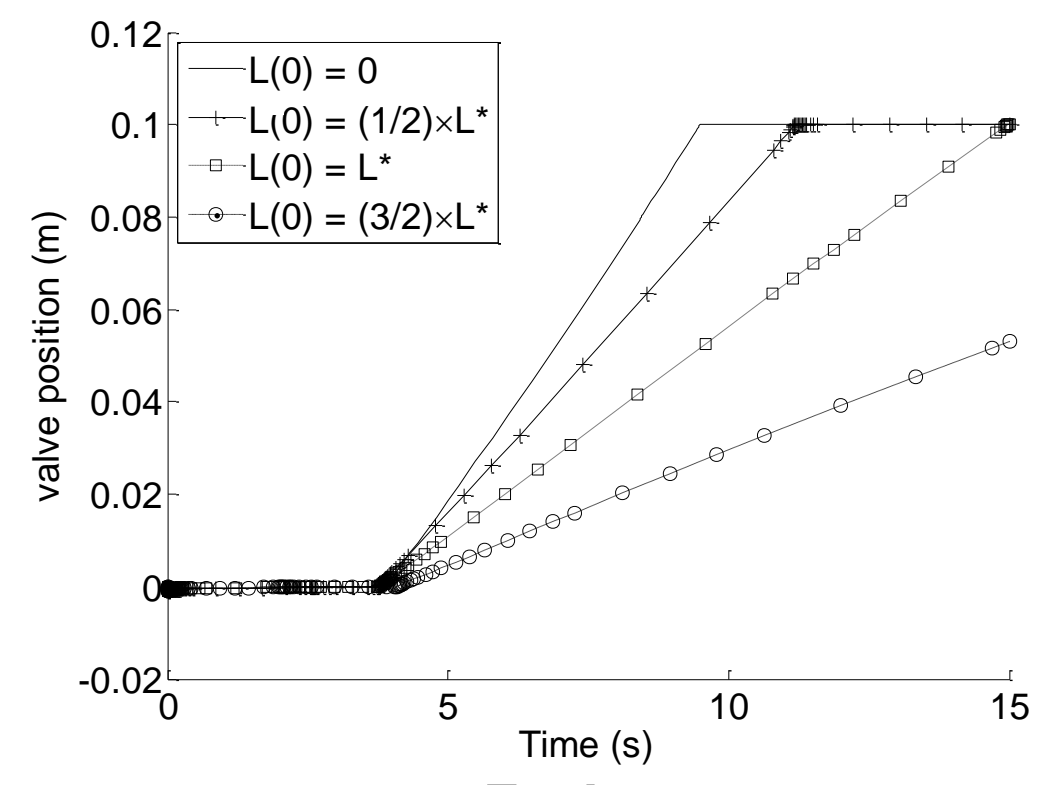

Fig. 4. Valve position for different sizes of the internal leakage.

\subsubsection{PDMP for MSPS considering dependence}

The degradation processes of the whole system are modeled by PDMP as follows:

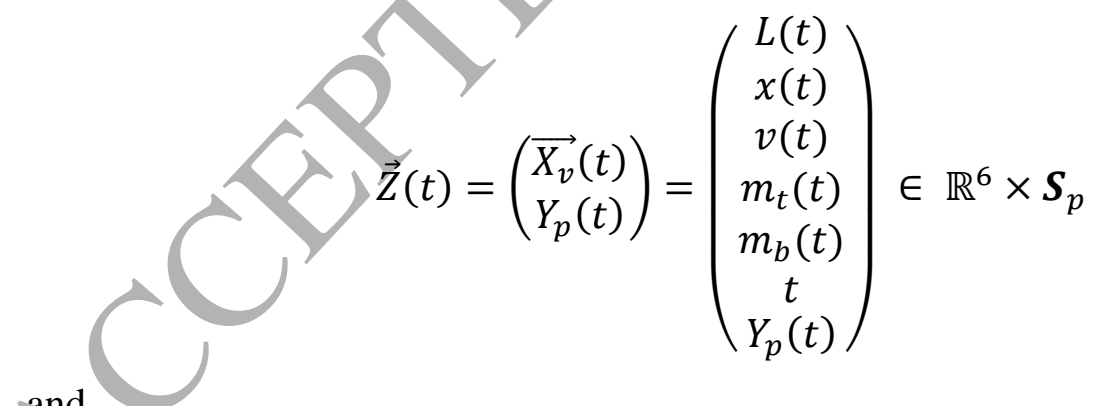

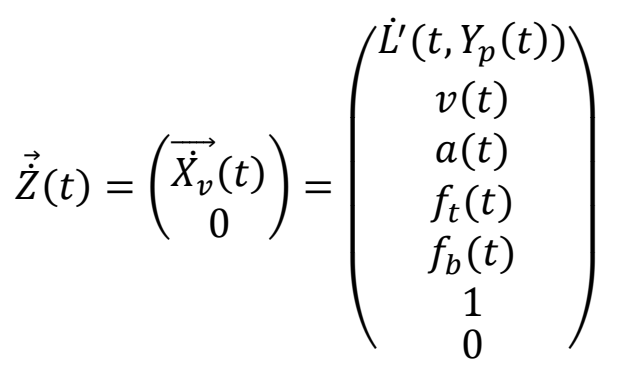

where $\dot{L}^{\prime}\left(t, Y_{p}(t)\right)$ is the derivative of the internal leakage of the valve, with 
consideration of the degradation dependence between the valve and the pump whereas the development of the internal leakage of the valve is dependent on the degradation state of the pump,

$$
\dot{L}^{\prime}\left(t, Y_{p}(t)\right)=w\left(1+\beta_{Y_{p}(t)}\right) r v(t)^{2}
$$

where $\beta_{Y_{p}(t)}$ is the relative increment of the developing rate of the internal leakage caused by the vibration of the pump (if we ignore the degradation dependence, then $\left.\beta_{Y_{p}(t)}=0\right)$. For illustrative purposes, we assume that $\beta_{3}=\beta_{0}=0, \beta_{2}=10 \%$ and $\beta_{1}=20 \%$. The times between two consequent jumps of PDMP/follow the exponential distribution with constant degradation transition rates of the pump. The space of the failure states of $\vec{Z}(t)$ is $\mathcal{F}=\mathbb{R}^{6} \times\{0\} \cup\left[L^{*},+\infty\right) \times \mathbb{R}^{5} \times \boldsymbol{S}_{p}$.

\subsubsection{Results and analysis}

Due to the large dimension of the PDMP and the complex formulation of the physic equations, the MC simulation method is adopted to solve the model.

The initial state of the system is as follows:

$$
\overrightarrow{Z^{\prime}}=\left(\begin{array}{c}
\overrightarrow{X_{v}}(0) \\
Y_{p}(0)
\end{array}\right)=\left(\begin{array}{c}
L(0)=0 \\
0 \\
0 \\
\frac{P_{s u p}\left(L_{s} A_{p}+V_{t 0}\right)}{R_{g} T} \\
\frac{P_{a t m} V_{b 0}}{R_{g} T} \\
0 \\
3
\end{array}\right)
$$

which means that the two components are both in perfect state and the valve is in the fully closed position. The command of the valve is a 30s-periodic-signal and the valve is commanded to open in the first half-period and to close in the second half. The pump is functioning until it reaches the failure state ' 0 '.

MC1, MC2 and MC3 are applied for the system reliability estimation over a time horizon of $T_{m i s s}=700 \mathrm{~s}$. The results are shown in Fig. 5, with the sample variances shown in Fig. 6. In order to appreciate the differences in the curves plotted in Fig. 5, the results between $460 \mathrm{~s}$ and $560 \mathrm{~s}$ are presented in Fig. 7. 


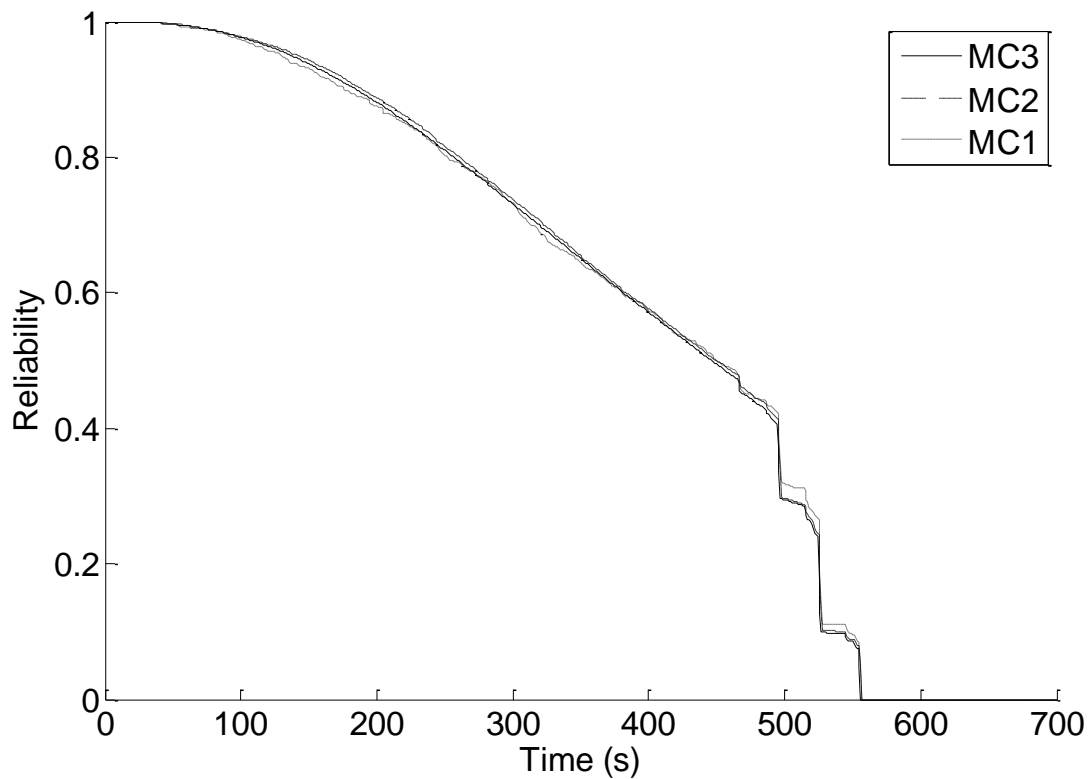

Fig. 5. System reliability obtained by $\mathrm{MC1}, \mathrm{MC} 2$ and $\mathrm{MC} 3$.

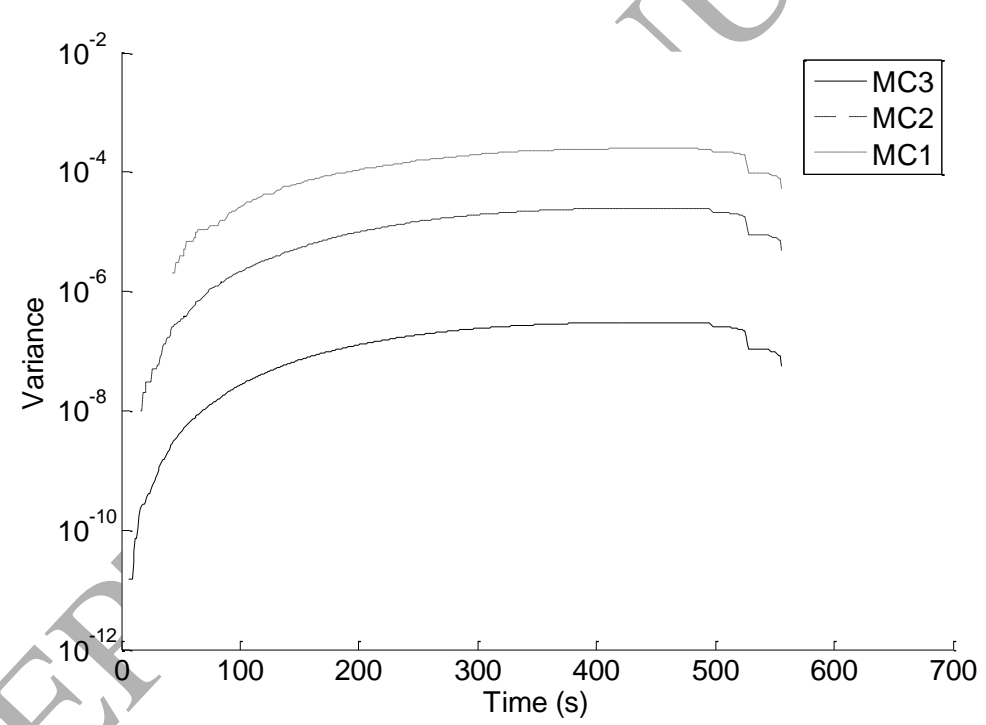

Fig. 6. Sample variances of system reliability obtained by MC1, MC2 and MC3. 


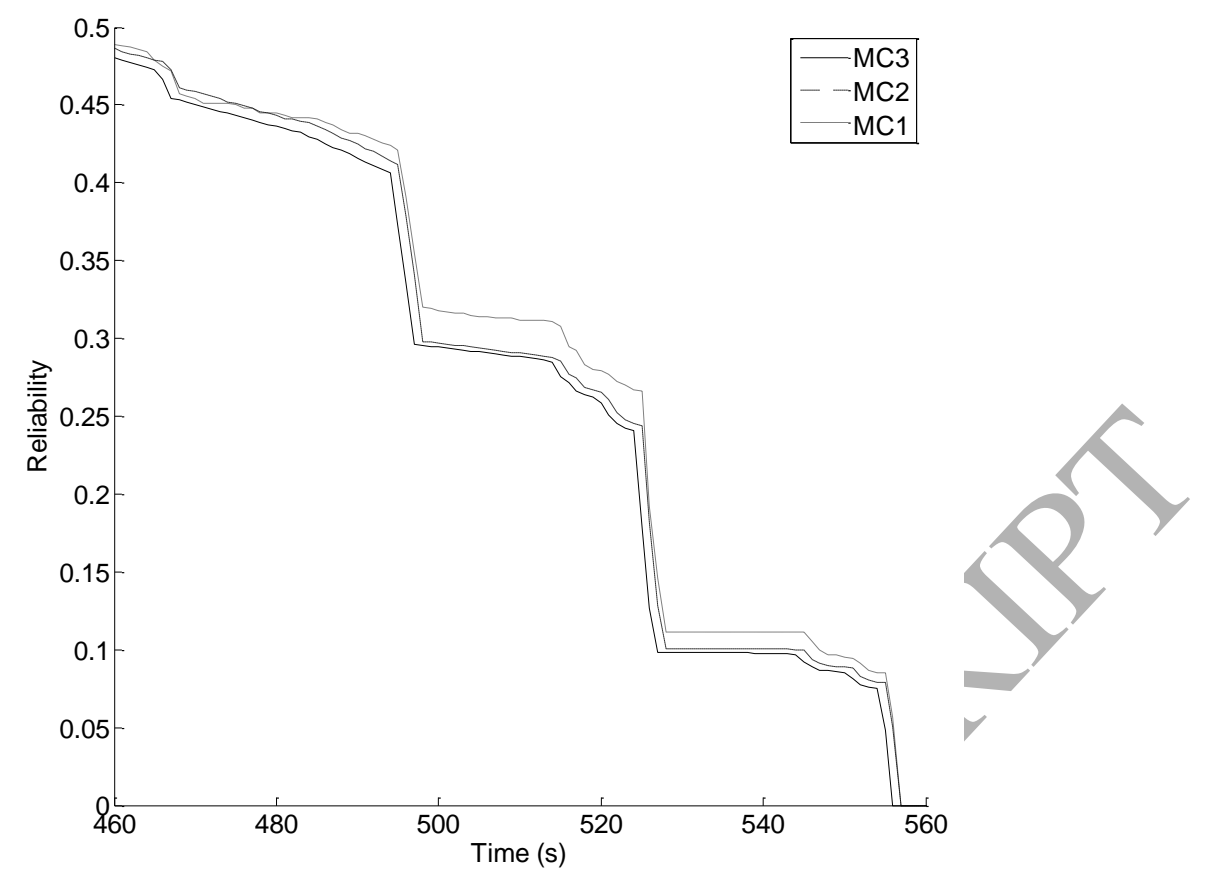

Fig. 7. System reliability with common degradation cause and degradation dependence obtained by MC1, MC2 and MC3 between $460 \mathrm{~s}$ and $560 \mathrm{~s}$.

In Fig. 8, we compare the system reliability with/without dependence, obtained by MC3. From the Figure, we can see that before $465.67 \mathrm{~s}$ (point A) the two curves coincide and the system reliability is equal to the reliability of the pump. After that time, valve failures begin to occur in some simulation trials, corresponding to realizations in which the pump jumps to state ' 1 ' very soon and stays there until the valve fails. The system reliability, then, experiences three sharp decreases at around $497.39 \mathrm{~s}$ (point B), 526.77 s (point C) and 556.45 s (point D) respectively, and the system is definitely failed afterwards. The longest failure time of the valve is at point $\mathrm{D}$, corresponding to the situation when the pump stays in the initial state ' 3 ' from the beginning until the failure of the valve. It is seen that neglecting degradation dependence might underestimate the system reliability. 


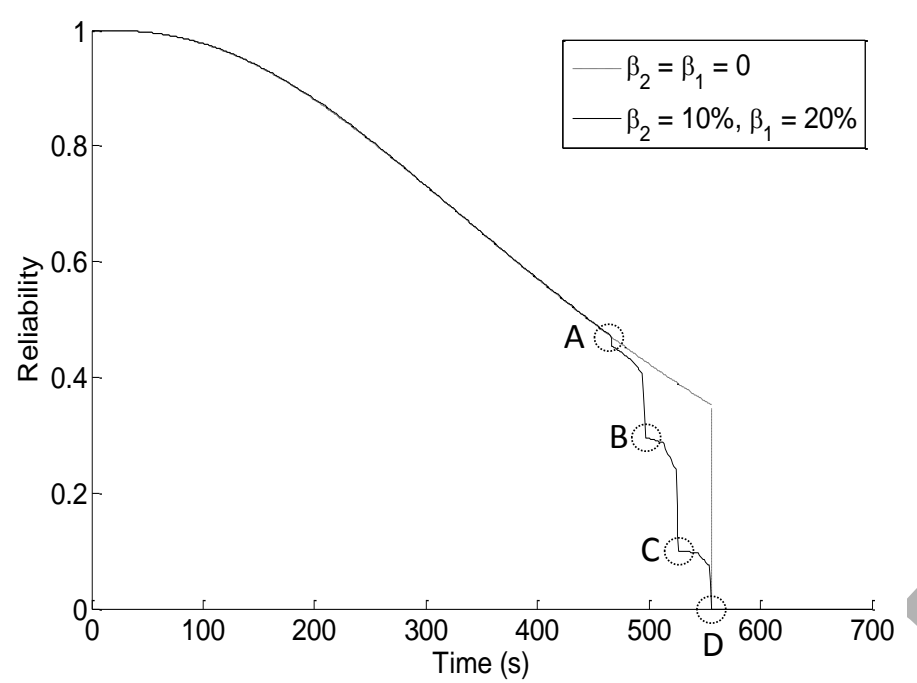

Fig. 8. System reliability with/without dependence.

\subsubsection{Case 2}

In this case study, the external leakage at the actuator connections to the bottom pneumatic port due to corrosion and other environmental factors is considered as relevant degradation mechanism, [8].

Let $D_{b}(t)$ denote the area of the leakage hole at the bottom pneumatic port at time $t$; the development of the leakage size is described by:

$$
\dot{D_{b}}(t)=\omega_{b}
$$

where $\omega_{b}=1 e-8 \mathrm{~m} 2 / \mathrm{s}$ is the original wear coefficient. The threshold of the area of the leakage hole can be calculated as $D_{b}^{*}=1.06 \mathrm{e}-5 \mathrm{~m}^{2}$ by using the same criteria given in Section 4.1 .

\subsubsection{PDMP for MSPS considering degradation dependence}

The degradation processes of the whole system are modeled by PDMP as follows:

and

$$
\vec{Z}(t)=\left(\begin{array}{c}
D_{b}(t) \\
Y_{p}(t)
\end{array}\right) \in \mathbb{R}^{+} \times \boldsymbol{S}_{p}
$$

$$
\overrightarrow{\dot{Z}}(t)=\left(\begin{array}{c}
\dot{D}_{b}^{\prime}(t) \\
0
\end{array}\right)=\left(\begin{array}{c}
\omega_{b}\left(1+\alpha_{Y_{p}(t)}\right) \\
0
\end{array}\right)
$$

where $\alpha_{Y_{p}(t)}$ is the relative increment of the developing rate of the external leakage at the bottom pneumatic port caused by the vibration of the pump at the degradation state ' 2 ' or ' 1 ' (if we ignore the degradation dependence, then $\alpha_{Y_{p}(t)}=0$ ). We assume that $\alpha_{3}=\alpha_{0}=0, \alpha_{2}=10 \%$ and $\alpha_{1}=20 \%$. The times between two consequent jumps of PDMP follow the exponential distribution with constant degradation transition rates of the pump. The space of the failure states of $\vec{Z}(t)$ is 
$\mathcal{F}=\mathbb{R}^{+} \times\{0\} \cup\left[D_{b}^{*},+\infty\right) \times \boldsymbol{S}_{p}$

The initial state of the system is assumed as follows:

$$
\overrightarrow{Z_{0}}=\left(\begin{array}{l}
D_{b}(0) \\
Y_{p}(0)
\end{array}\right)=\left(\begin{array}{l}
0 \\
3
\end{array}\right)
$$

which means that the two components are both in their perfect state.

\subsubsection{Results and analysis}

MC simulation method and FV scheme are applied for the estimation of the system reliability over a time horizon of $T_{\text {miss }}=1000 \mathrm{~s}$. The results obtained by MC1, MC2 and MC3 are shown in Fig. 9.

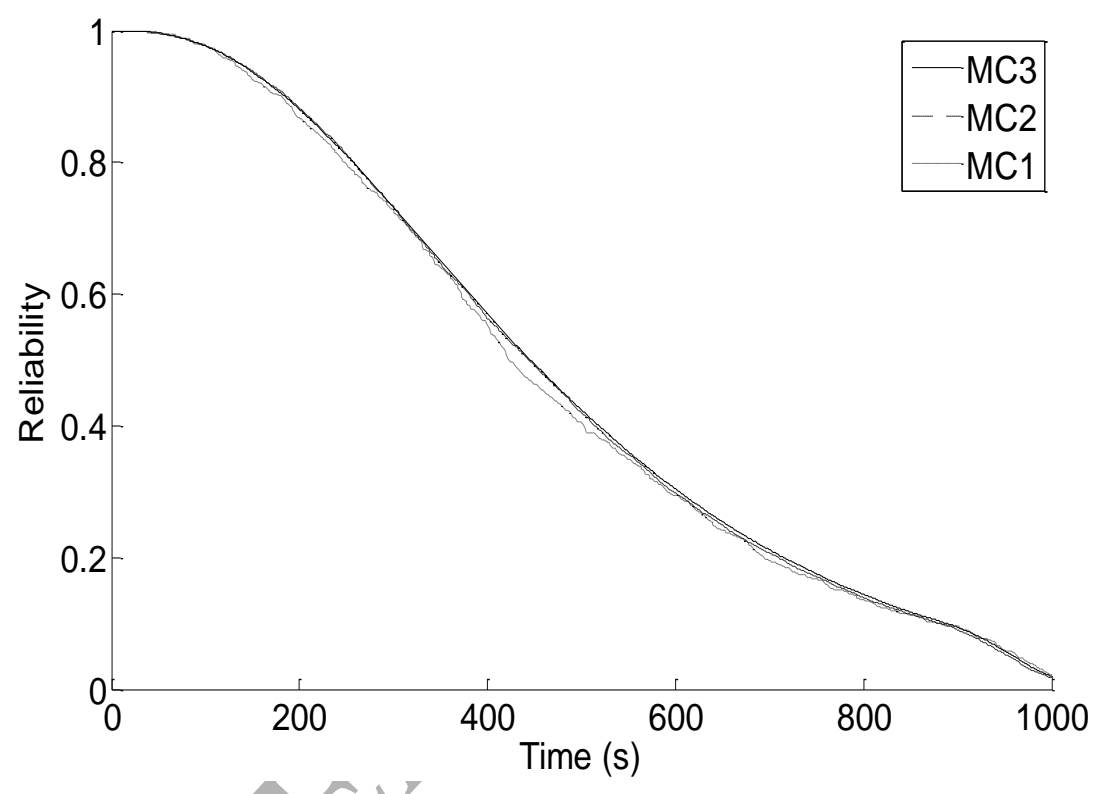

Fig. 9. System reliability obtained by MC1, MC2 and MC3.

For the EV scheme, the state space $\mathbb{R}^{+}$of $D_{b}(t)$ has been divided into an admissible mesh $\mathcal{M}=\bigcup_{n=0,1,2, \ldots}\left[n \Delta x,(n+1) \Delta x\left[\right.\right.$ and the time space $\mathbb{R}^{+}$has been divided into small intervals $\mathbb{R}^{+}=\bigcup_{n=0,1,2, \ldots}[n \Delta t,(n+1) \Delta t[$. The values of space step $\Delta x$ and time step $\Delta t$ can influence the accuracy of the results. We have considered 7 different parameter settings: (1) FV1: $\Delta x=1 e-8, \Delta t=1$; (2) FV2: $\Delta x=5 e-8, \Delta t=1 ;$ (3) FV2a: $\Delta x=10 e-8, \Delta t=1$; (4) FV3: $\Delta x=1 e-$ 8, $\Delta t=5$, (5) FV3a: $\Delta x=1 e-8, \Delta t=10$, (6) FV4: $\Delta x=5 e-8, \Delta t=5$ and (7) FV5: $\Delta x=10 e-8, \Delta t=10$. Their results are shown in Fig. 9-12.

We compare the results obtained by FV1 and MC3 in Fig. 10, where it is shown that FV scheme can lead to results comparable to those of the MC simulation method. The effect of variations in $\Delta x$ is studied in Fig. 11, where it can be seen that before around $730 \mathrm{~s}$ (point A) the three curves match. Up to that time, the system reliability is equal to the reliability of the pump. After that time, $D_{b}(t)$ approaches the threshold $D_{b}^{*}$ and valve failure begins to occur, so that the effect of variations in $\Delta x$ becomes more distinct since smaller $\Delta x$ leads to more accurate estimation of $D_{b}(t)$ 
and, thus, more accurate estimation of the system reliability. The effects of variation in $\Delta t$ is studied in Fig. 12, where we can see that the effect of variations in $\Delta t$ is visible from the beginning, since $\Delta t$ can influence the estimation of both $D_{b}(t)$ and $Y_{p}(t)$ and, thus, influence the estimation of the system reliability from the beginning. The joint effect of variations in $\Delta x$ and $\Delta t$ is shown in Fig. 13.

In general cases, the appropriate value of the space step $\Delta x$ is problem-specific and can only be approximated since no analytical expression is available due to the complexity of PDMP model. In this work, the approximation method starts at assigning an initial value to $\mathrm{A}$ and setting a value to the ratio $\mathrm{B}(>1)$, by the experimenter. The initial value of $A$ is not chosen as small as possible, since it may be significantly different from the appropriate value of the space step $\Delta x$. Besides, a very large value of the ratio $B$ may make it difficult to find the appropriate value of the space step $\Delta x$ if it is around the value of $A$. According to our experiments, the initial value of $A$ can be chosen between $1 \%$ and $10 \%$ of the failure threshold value and the ratio $B$ can be chosen between 2 and 10. Their values are problem-specific and can be adjusted with respect to the performance of the approximation method.

If the difference between the results obtained by setting $\Delta x=A$ and those by setting $\Delta x=A / B$ is negligible (if the absolute relative difference of the two results is smaller than $r \%$, e.g. $r=1$, which is chosen by the experimenter according to the accuracy requirement of the problem under study, it can be considered as negligible.), then we replace $A$ by $A \cdot B$ and redo the comparison. This procedure is repeated until the difference is not negligible. Then, $A$ is assigned to $\Delta x$. On the contrary, if the difference between the results obtained by setting $\Delta x=A$ and those by setting $\Delta x=A / B$ is not negligible, we replace $A$ by $A / B$ and redo the comparison. This procedure is repeated until the difference is negligible. Then, $A$ is assigned to $\Delta x$. Usually, the order of magnitude of the value of $\Delta x$ is close to that of the growth rate of the continuous degradation variable since the evolution of the continuous degradation variable can, then, be well approximated by using grids of size $\Delta x$. In the case study, the value of $\Delta x$ in FV1 is chosen as $1 e-8$ by using the above described method given that the growth rate of the leak size at the bottom pneumatic port $\omega_{b}=1 e-8 \mathrm{~m} 2 / \mathrm{s}$.

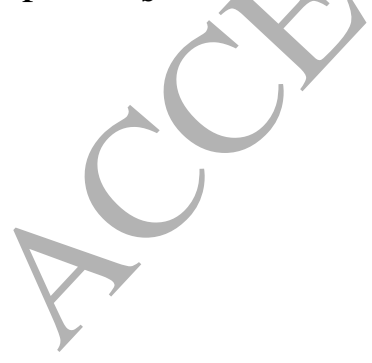




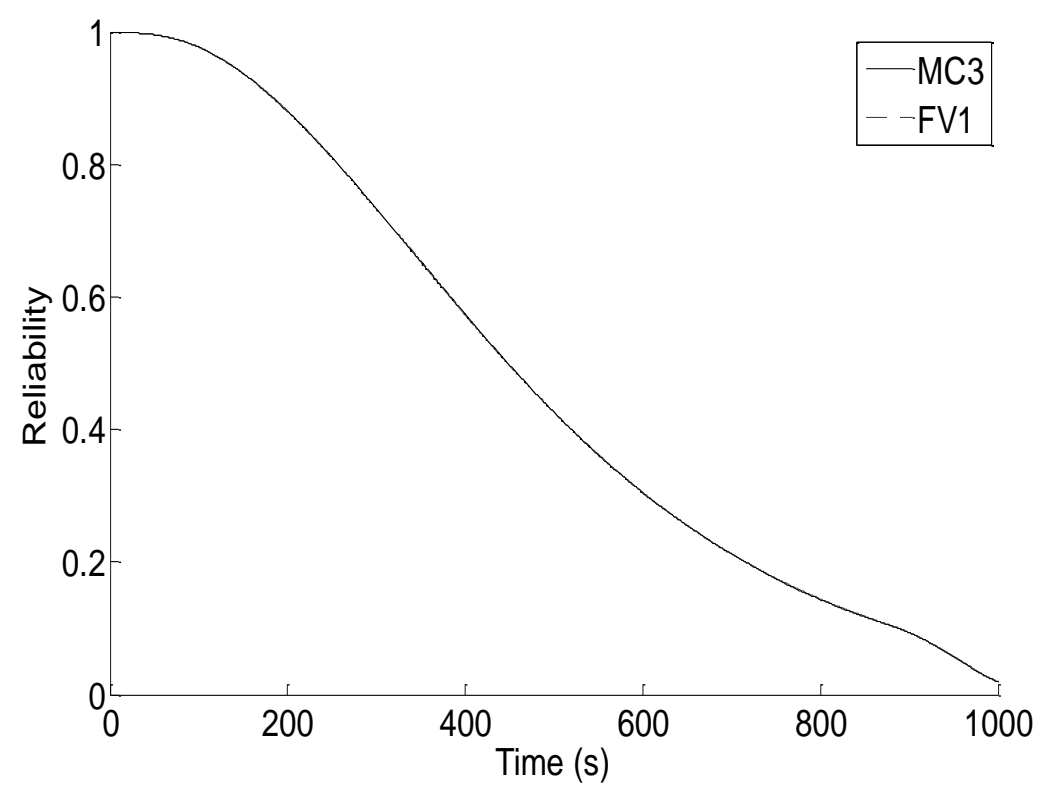

Fig. 10. System reliability obtained by FV1 and MC3.

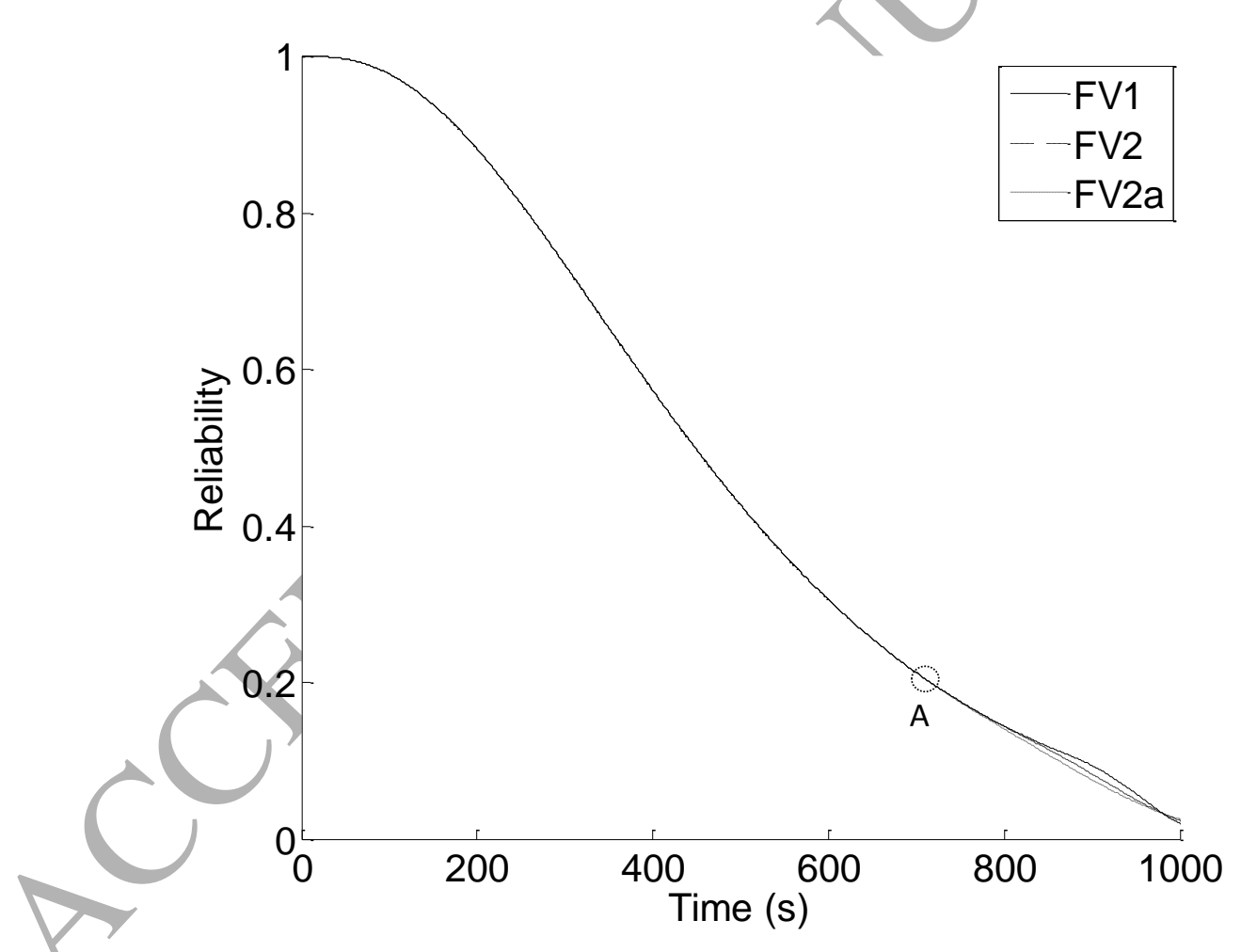

Fig. 11. System reliability obtained by FV1, FV2 and FV2a. 


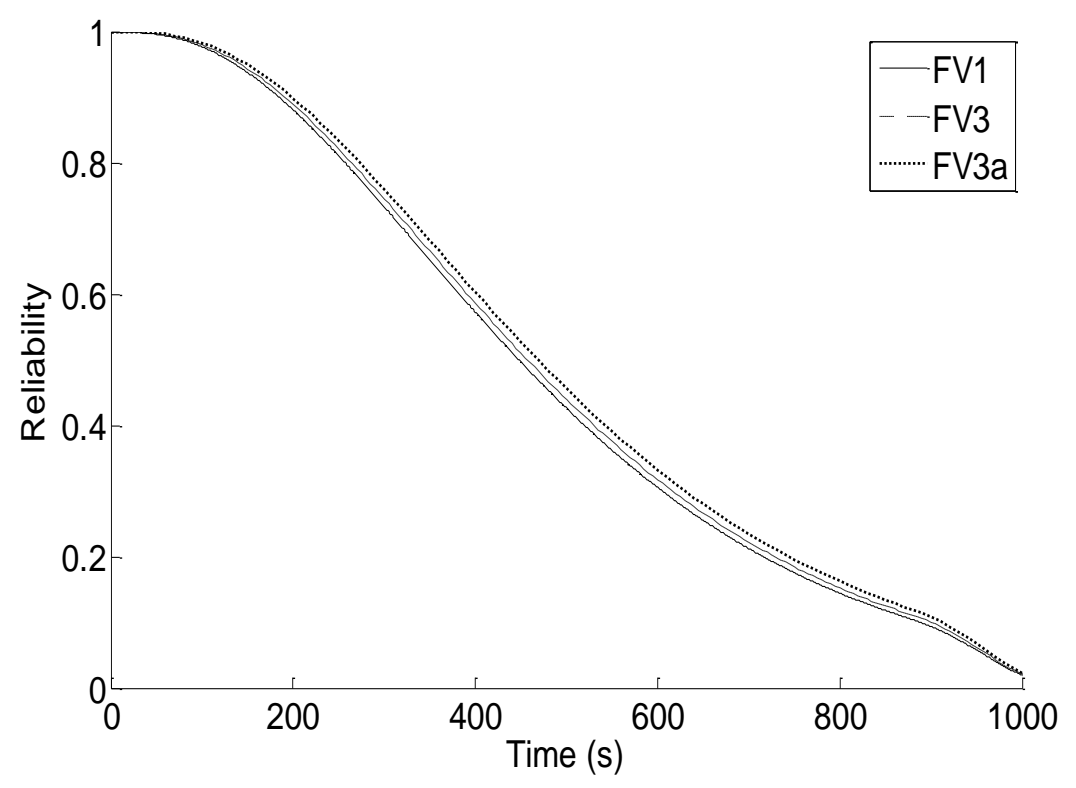

Fig. 12. System reliability obtained by FV1, FV3 and FV3a.

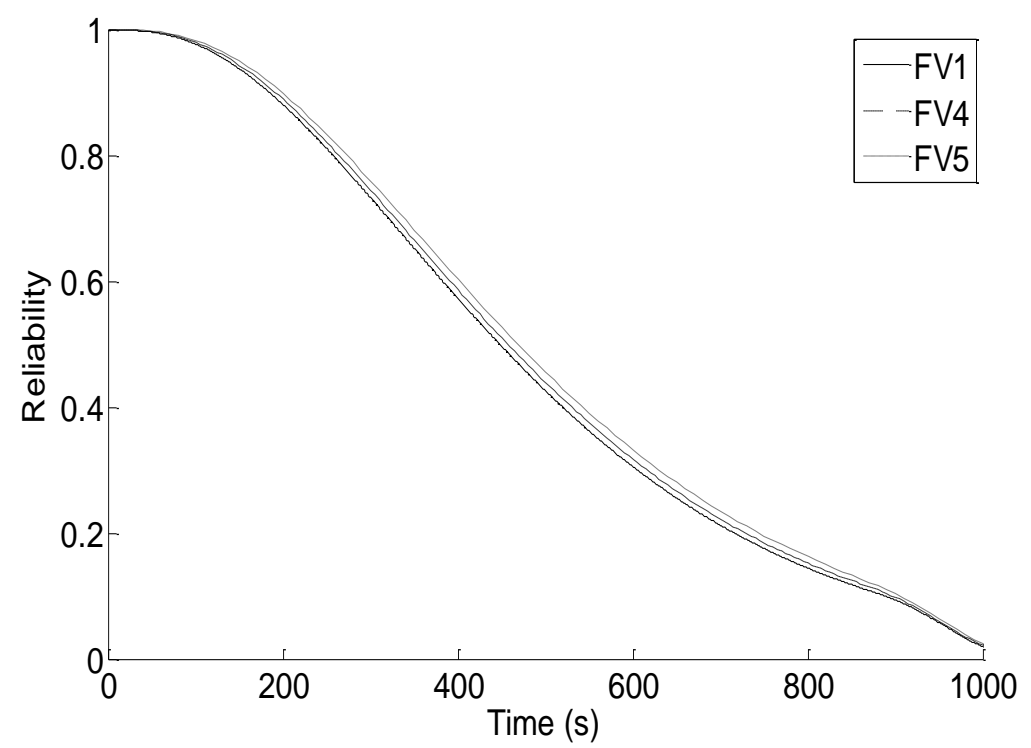

Fig. 13. System reliability obtained by FV1, FV4 and FV5.

\subsection{Comparisons}

The numerical comparisons of the two methods are reported in Table III. With reference to the results obtained by MC3, as expected, the accuracy of MC simulation methods increases as the number of replications is increased, while that of FV scheme is dependent on the space step $\Delta x$ and the time step $\Delta t$. The accuracy of FV scheme can be improved by reducing $\Delta x$ and $\Delta t$ individually or jointly. The variation in $\Delta t$ can influence the system reliability estimations over the entire mission time since $\Delta t$ 
determines the estimated states of all degradation processes. However, the variation in $\Delta x$ may influence the system reliability estimations after certain time if the system failures at early stage are only due to certain degradation processes modeled by MSMs, of which the estimated states are independent of $\Delta x$. By properly choosing the step sizes, FV scheme can lead to results comparable to those of the MC simulation. The average computation time of the two methods shows that the FV scheme is more efficient than MC simulation for simple and low dimensional problems. However, it should be noted that the memory requirement of the FV scheme is much higher than that of MC simulation method and the FV scheme is sensitive to the space step and time step. The computational expenses of the MC simulation method increase linearly as the number of replications increases and that of $\mathrm{FV}$ scheme is almost linear with $\Delta x \cdot \Delta t$.

Table III Comparisons of the system reliability results obtained by MC símulation method and FV scheme

\begin{tabular}{|c|c|c|c|c|c|}
\hline \multicolumn{2}{|c|}{ Methods } & \multirow{2}{*}{$\begin{array}{c}\text { System } \\
\text { reliability } \\
\text { at } 1000 \mathrm{~s} \\
\mathbf{0 . 0 1 9 7}\end{array}$} & \multirow{2}{*}{$\begin{array}{c}\text { Relative } \\
\text { change } \\
\text { with } \\
\text { respect to } \\
\text { MC3 }\end{array}$} & \multirow{2}{*}{$\begin{array}{l}\text { Average } \\
\text { computation } \\
\text { time }(\mathrm{s}) \\
\\
1.41\end{array}$} & \multirow{2}{*}{$\begin{array}{c}\text { Memory } \\
\text { consumption } \\
(\mathrm{KB}) \\
\\
8.17\end{array}$} \\
\hline $\mathrm{MC}$ & MC3 & & & & \\
\hline simulation & $\mathrm{MC} 2$ & 0.0175 & $11.17 \%$ & 0.14 & 8.17 \\
\hline method & MC1 & 0.023 & $16.75 \%$ & 0.014 & 8.17 \\
\hline \multirow{7}{*}{$\begin{array}{c}\text { FV } \\
\text { scheme }\end{array}$} & FV1 & 0.0199 & $1.02 \%$ & 0.17 & 33.62 \\
\hline & FV2 & 0.0237 & $20.30 \%$ & 0.042 & 13.26 \\
\hline & FV2a & 0.0253 & $28.43 \%$ & 0.021 & 10.72 \\
\hline & $\mathrm{FV} 3$ & 0.0212 & $7.61 \%$ & 0.033 & 27.22 \\
\hline & $\mathrm{FV} 3 \mathrm{a}$ & 0.0231 & $17.26 \%$ & 0.017 & 26.41 \\
\hline & FV4 & 0.0218 & $10.66 \%$ & 0.0058 & 6.86 \\
\hline & FV5 & 0.0241 & $22.34 \%$ & 0.00027 & 3.51 \\
\hline
\end{tabular}

4.5. Guidelines for the selection of the MC simulation method and FV scheme

Table IV summarizes the qualitative insights drawn from the comparative studies of the two numerical approaches.

Table IV Comparisons of the two numerical approaches

\begin{tabular}{|c|c|c|}
\hline & MC simulation method & FV scheme \\
\hline Parameters & Number of replications & Space step, Time step \\
\hline Accuracy & Medium & High \\
\hline Computation time & Long & Short \\
\hline Memory consumption & Low & High \\
\hline Efficiency & Low & High \\
\hline Scope of application & Large & Small \\
\hline
\end{tabular}




\begin{tabular}{|l|c|c|}
\hline Ease of Implementation & Yes & Generally no \\
\hline
\end{tabular}

The MC simulation method requires a number of replications to achieve a desired level of accuracy, whereas the FV scheme needs to discretize the time space and state space by properly choosing the corresponding step sizes. Due to the discretization, the memory consumption of FV scheme is typically larger than that of the MC simulation method. The MC simulation method is easy to be implemented by the practitioners without restrictions on the dimension of the problem, like for PDMP. In reverse, the price to pay is that the MC simulation method can be quite time-consuming. The FV scheme is an alternative that appears to be efficient and lead to results comparable to those of the MC simulation method with acceptable computing time. However, it is unsuited for high-dimensional problems or problems with complex equations describing the deterministic evolution, and it is also relatively difficult to implement and deploy.

Given the above observations, the following guidelines for selection may be helpful:

- For high dimensional problems or problems with complex equations describing the deterministic evolution, the MC simulation method is preferred.

- For low dimensional problems or problems with simple equations describing the deterministic evolution, the FV scheme is preferred. Note that in some cases the high dimensional problem can be decomposed into several low dimensional ones mutually independent on each other. Then, the FV schemes can be run on low dimensional problems in parallel.

\section{CONCLUSIONS}

PDMP approach can be employed to model MSPS subject to degradation dependence. The significance of the method lies in the possibility that it offers to describe the degradation dependence between PBMs, between MSMs and between the two types of models. MC simulation method and FV scheme are two widely used approaches for the system reliability assessment based on the PDMP. In the present work, a comparative study of the two approaches has been carried out to study their accuracy, efficiency, memory requirement, scope of application and ease of implementation and clear guidelines for their selection have been provided. Results show that the MC simulation method is easy to be implemented and has wide applicability, since it has no restriction on the dimension of the underlying PDMP modeling the degradation processes. The FV scheme, although relatively difficult to handle and more demanding in terms of computer memory, is computationally more efficient and can lead to results comparable to those of the MC simulation method for simple and low dimensional problems. The MC simulation method requires a number of replications to achieve a desired level of accuracy, since the accuracy of numerical solutions of the ordinary differential equations can be controlled. The FV scheme needs to discretize the time space and state space by properly choosing the corresponding step sizes. 
As future research, we plan to study acceleration techniques for the MC simulation method, to relieve the computational burden.

\section{Acknowledgments}

The authors would like to thank the anonymous referees for their constructive comments. Professor Li's work was partially supported by the National Natural Science Foundation of China under a key project grant No. 71731008.

\section{References}

[1] Y.-F. Li, E. Zio, and Y.-H. Lin, "A Multistate Physics Model of Component Degradation Based on Stochastic Petri Nets and Simulation," Reliability, IEEE Transactions on, vol. 61, pp. 921 - 931 , 2012.

[2] O. Chryssaphinou, N. Limnios, and S. Malefaki, "Multi-state reliability systems under discrete time semi-Markovian hypothesis," Reliability, IEEE Transactions on, vol. 60, pp. 80-87, 2011.

[3] M. Giorgio, M. Guida, and G. Pulcini, "An age-and state-dependent Markov model for degradation processes," IIE Transactions, vol. 43, pp. 621-632, 2011.

[4] W. Li and H. Pham, "Reliability modeling of multi-state degraded systems with multi-competing failures and random shocks," Reliability, IEEE Transactions on, vol. 54, pp. 297-303, 2005.

[5] A. Lisnianski and G. Levitin, Multi-state system reliability: assessment, optimization and applications: World scientific, 2003.

[6] Y. Ding, M. J. Zuo, A. Lisnianski, and Z. Tian, "Fuzzy multi-state systems: general definitions, and performance assessment," Reliability, IEEE Transactions on, vol. 57, pp. 589-594, 2008.

[7] R. Moghaddass and M. J. Zuo, "Multistate Degradation and Supervised Estimation Methods for a Condition-Monitored Device," IIE Transactions, vol. 46, pp. 131-148, 2014.

[8] M. Daigle and K. Goebel, "A model-based prognostics approach applied to pneumatic valves," International Journal of Prognostics and Health Management, vol. 2, pp. 1-16, 2011.

[9] M. Daigle and K. Goebel, "Multiple damage progression paths in model-based prognostics," in Aerospace Conference, 2011 IEEE, 2011, pp. 1-10.

[10] E. Keedy and Q. Feng, "A physics-of-failure based reliability and maintenance modeling framework for stent deployment and operation," Reliability Engineering \& System Safety, vol. 103, pp. 94-101, 2012.

[11] S. Reggiani, S. Poli, M. Denison, E. Gnani, A. Gnudi, G. Baccarani, et al., "Physics-Based Analytical Model for HCS Degradation in STI-LDMOS Transistors," Electron Devices, IEEE Transactions on, vol. 58, pp. 3072-3080, 2011.

[12] M. Chookah, M. Nuhi, and M. Modarres, "A probabilistic physics-of-failure model for prognostic health management of structures subject to pitting and corrosion-fatigue," Reliability Engineering \& System Safety, vol. 96, pp. 1601-1610, 2011.

[13] N. Rasmekomen and A. K. Parlikad, "Maintenance Optimization for Asset Systems With Dependent Performance Degradation," Reliability, IEEE Transactions on, vol. 62, pp. 362 - 367 , 2013.

[14] S. Song, D. W. Coit, Q. Feng, and H. Peng, "Reliability analysis for multi-component systems subject to multiple dependent competing failure processes," Reliability, IEEE Transactions on, vol. 63, pp. 331-345. 
[15] H. Peng, Q. Feng, and D. W. Coit, "Reliability and maintenance modeling for systems subject to multiple dependent competing failure processes," IIE transactions, vol. 43, pp. 12-22, 2010.

[16] Y. Wang and H. Pham, "Modeling the dependent competing risks with multiple degradation processes and random shock using time-varying copulas," Reliability, IEEE Transactions on, vol. 61, pp. 13-22, 2012.

[17] Q. Yang, N. Zhang, and Y. Hong, "Reliability Analysis of Repairable Systems With Dependent Component Failures Under Partially Perfect Repair," Reliability, IEEE Transactions on, vol. 62, pp. 490-498, 2013.

[18] D. Straub, "Stochastic modeling of deterioration processes through dynamic Bayesian networks," Journal of Engineering Mechanics, vol. 135, pp. 1089-1099, 2009.

[19] Y.-H. Lin, Y. Li, and E. Zio, "Dynamic Reliability Models for Multiple Dependent Competing Degradation Processes," Proceedings of ESREL 2014, pp. 775-782, 2014.

[20] M. H. Davis, Markov models and optimization vol. 49: CRC Press, 1993.

[21] M. H. Davis, "Piecewise-deterministic Markov processes: A general class of non-diffusion stochastic models," Journal of the Royal Statistical Society. Series B (Methodological), pp. 353-388, 1984.

[22] M. Jacobsen, Point process theory and applications: marked point and piecewise deterministic processes: Springer, 2006.

[23] C. Cocozza-Thivent, "Processus de renouvellement markovien, Processus de Markov déterministes par morceaux," Online book available on the webpage: http://perso-math. univ-mlv. fr/users/cocozza. christiane/recherche-pageperso/PresentationRMetPDMP. html, 2011.

[24] M. Marseguerra and E. Zio, "Monte Carlo approach to PSA for dynamic process systems," Reliability Engineering \& System Safety, vol. 52, pp. 227-241, 1996.

[25] J. Chiquet, N. Limnios, and M. Eid, "Piecewise deterministic Markov processes applied to fatigue crack growth modelling," Journal of Statistical Planning and Inference, vol. 139, pp. 1657-1667, 2009.

[26] H. Zhang, F. Innal, F. Dufour, and Y. Dutuit, "Piecewise Deterministic Markov Processes based approach applied to an offshore oil production system," Reliability Engineering \& System Safety, vol. 126, pp. 126-134, 6// 2014.

[27] H. Zhang, F. Innal, F. o. Dufour, and Y. Dutuit, "Piecewise Deterministic Markov Processes based approach applied to an offshore oil production system," Reliability Engineering \& System Safety, vol. 126, pp. 126-134.

[28] W. Lair, S. Mercier, M. Roussignol, and R. Ziani, "Piecewise deterministic Markov processes and maintenance modeling: application to maintenance of a train air-conditioning system," Proceedings of the Institution of Mechanical Engineers, Part O: Journal of Risk and Reliability, vol. 225, pp. 199-209, 2011.

[29] C. Cocozza-Thivent, R. Eymard, and S. Mercier, "A finite-volume scheme for dynamic reliability models," IMA journal of numerical analysis, vol. 26, pp. 446-471, 2006.

[30] R. Eymard, S. Mercier, and A. Prignet, "An implicit finite volume scheme for a scalar hyperbolic problem with measure data related to piecewise deterministic Markov processes," journal of Computational and Applied Mathematics, vol. 222, pp. 293-323, 2008.

[31] Y.-H. Lin, Y.-F. Li, and E. Zio, "Fuzzy reliability assessment of systems with multiple-dependent 
competing degradation processes," Fuzzy Systems, IEEE Transactions on, vol. 23, pp. 1428-1438, 2015.

[32] R. Eymard, S. Mercier, A. Prignet, and M. Roussignol, "A finite volume scheme for sensitivity analysis in dynamic reliability," Numerische Mathematik, vol. 104, pp. 457-488, 2008.

[33] Y. H. Lin, Y. F. Li, and E. Zio, "A Reliability Assessment Framework for Systems With Degradation Dependency by Combining Binary Decision Diagrams and Monte Carlo Simulation," IEEE Transactions on Systems Man \& Cybernetics Systems, pp. 1-9, 2015.

[34] E. Lewis and F. Böhm, "Monte Carlo simulation of Markov unreliability models," Nuclear Engineering and Design, vol. 77, pp. 49-62, 1984.

[35] E. Hairer, S. P. Nørsett, and G. Wanner, Solving Ordinary Differential Equations I: Nonstiff Problems: Springer, 1993.

[36] E. Hairer, S. P. Nørsett, and G. Wanner, Solving Ordinary Differential Equations II: Stiff and Differential-Algebraic Problems: Springer, 1996.

[37] R. Coudray and J. Mattei, "System reliability: An example of nuclear reactor system analysis," Reliability Engineering, vol. 7, pp. 89-121, 1984.

[38] S. Zhang, M. Hodkiewicz, L. Ma, and J. Mathew, "Machinery condition prognosis using multivariate analysis," in Engineering Asset Management, ed: Springer, 2006, pp. 847-854.

[39] P. Moussou, S. Cambier, D. Lachene, S. Longarini, L. Paulhiac, and V. Villouvier, "Vibration investigation of a French PWR power plant piping system caused by cavitating butterfly valves," ASME-PUBLICATIONS-PVP, vol. 420, pp. 99-106, 2001.

[40] P. Vlok, J. Coetzee, D. Banjevic, A. Jardine, and V. Makis, "Optimal component replacement decisions using vibration monitoring and the proportional-hazards model," Journal of the operational research society, pp. 193-202, 2002.

[41] M. Daigle and K. Goebel, "Model-based prognostics under limited sensing," in Aerospace Conference, 2010 IEEE, 2010, pp. 1-12.

[42] A. Lorton, M. Fouladirad, and A. Grall, "Computation of remaining useful life on a physic-based model and impact of a prognosis on the maintenance process," Proceedings of the Institution of Mechanical Engineers, Part O: Journal of Risk and Reliability, 2013.

\section{Appendix A: MC simulation method}

To apply the MC simulation method, eq. (4) is written as follows:

$$
\begin{gathered}
P\left[\overrightarrow{Z_{n+1}} \in B, T_{n+1} \in\left[T_{n}, T_{n}+\Delta t\right] \mid \overrightarrow{Z_{n}}=\vec{\imath}, \boldsymbol{\theta}_{\boldsymbol{K}}\right] \\
=\iint_{B *[0, \Delta t]} N\left(\vec{\imath}, \overrightarrow{d z}, d s \mid \boldsymbol{\theta}_{\boldsymbol{K}}\right) \\
\forall n \geq 0, \Delta t \geq 0, \vec{\imath} \in \boldsymbol{E}, B \in \varepsilon
\end{gathered}
$$

where $\varepsilon$ is a $\sigma$-algebra of $\boldsymbol{E}[23]$ and $N\left(\vec{l}, \overrightarrow{d z}, d s \mid \boldsymbol{\theta}_{\boldsymbol{K}}\right)$ is a semi-Markov kernel on $\boldsymbol{E}$, which verifies that $\iint_{\boldsymbol{E} *[0, \Delta t]} N\left(\vec{\imath}, \overrightarrow{d z}, d s \mid \boldsymbol{\theta}_{\boldsymbol{K}}\right) \leq 1, \forall \Delta t \geq 0, \vec{\imath} \in \boldsymbol{E}$. It can be 
further developed as:

$$
N\left(\vec{l}, \overrightarrow{d z}, d s \mid \boldsymbol{\theta}_{\boldsymbol{K}}\right)=d F_{\vec{\imath}}\left(s \mid \boldsymbol{\theta}_{\boldsymbol{K}}\right) \beta\left(\vec{l}, s, \overrightarrow{d z} \mid \boldsymbol{\theta}_{\boldsymbol{K}}\right)
$$

where

$$
d F_{\vec{\imath}}\left(s \mid \boldsymbol{\theta}_{\boldsymbol{K}}\right)
$$

is the probability density function of $T_{n+1}-T_{n}$ given $\overrightarrow{Z_{n}}=\vec{\imath}$ and

$$
\beta\left(\vec{l}, s, \overrightarrow{d z} \mid \boldsymbol{\theta}_{\boldsymbol{K}}\right)
$$

is the conditional probability of state $\overrightarrow{Z_{n+1}}$ given $T_{n+1}-T_{n}=s$.

Then, the MC simulation method can be used to estimate the reliability of the system within a certain mission time $T_{m i s s}$, given the initial system state $\overrightarrow{Z_{0}}$ at time $T_{0}=0$. The method to simulate the behavior of the system consists in sampling the transition time from eq. (26) and the arrival state from eq. (27) for the components in the second group and, then, using the physics eq. (3) to calculate the evolution of the components in the first group within the transition times. Each simulation trial continues until the time of system evolution reaches $T_{\text {miss }}$ or until the system enters the failure space $\mathcal{F}$, event whose occurrence is recorded for the statistical estimation of the system reliability.

\section{The simulation procedure}

The procedure of the MC simulation method [33] is as follows:

Set $N_{\max }$ (the maximum number of replications) and $k=0$ (index of MC trials)

Set $k^{\prime}=0$ (number of MC trials that end in failure state)

While $k<N_{\max }$

Initialize the system by setting $\overrightarrow{Z^{\prime}}=\left(\begin{array}{c}\vec{X}(0) \\ \vec{Y}\end{array}\right)$ (initial system state) and the time $T=0$ (initial system time)

Set $t^{\prime}=0$ (state holding time)

While $T<T_{\text {miss }}$

Sample a $t^{\prime}$ by using the probability density function eq. (26)

Sample an arrival state $\overrightarrow{Y^{\prime}}$ for stochastic process $\vec{Y}(t)$ from all possible states, by using the conditional probability function eq. (27)

Set $T=T+t^{\prime}$

Calculate $\vec{X}(t)$ in the interval $\left[T-t^{\prime}, T\right]$ by using the physics equations eq. (3)

Set $\overrightarrow{Z^{\prime}}=\left(\begin{array}{c}\vec{X}(T) \\ \overrightarrow{Y^{\prime}}\end{array}\right)$ 
If $T \leq T_{\text {miss }}$

$$
\text { If } \exists t \in\left[T-t^{\prime}, T\right], \vec{Z}(t)=\left(\begin{array}{c}
\vec{X}(t) \\
\vec{Y}
\end{array}\right) \in \mathcal{F}
$$

Set $k^{\prime}=k^{\prime}+1$

Break

\section{End if}

Else (when $T>T_{\text {miss }}$ )

$$
\text { If } \exists t \in\left[T-t^{\prime}, T_{\text {miss }}\right], \vec{Z}(t)=\left(\begin{array}{c}
\vec{X}(t) \\
\vec{Y}
\end{array}\right) \in \mathcal{F}
$$

Set $k^{\prime}=k^{\prime}+1$

\section{Break}

\section{End if}

\section{End if}

Set $\vec{Y}=\overrightarrow{Y^{\prime}}$

\section{End While}

Set $k=k+1$

\section{End While $\square$}

The estimated component reliability at time $T_{\text {miss }}$ can be obtained by

$$
\hat{R}\left(T_{\text {miss }}\right)=1-k^{\prime} / N_{\max }
$$

where $k^{\prime}$ represents the number of trials that end in the failure state of the system and the sample variance is [34]:

$$
\operatorname{var}_{\hat{R}\left(T_{\text {miss }}\right)}=\hat{R}\left(T_{\text {miss }}\right)\left(1-\hat{R}\left(T_{\text {miss }}\right)\right) /\left(N_{\text {max }}-1\right)
$$

To calculate the value of $\vec{X}(t)$, Runge-Kutta methods [35, 36] can be applied for the numerical solution of the ordinary differential equations, which can guarantee the convergence of the numerical methods with accuracy of certain order by controlling the local truncation error. Therefore, higher number of replications will lead to lower sample variance in MC simulation method.

\section{Appendix B: FV method}

\section{Assumptions}

This approach can be applied under the following assumptions:

- The transition rates $\lambda_{\vec{\imath}}\left(\vec{J} \mid ;, \boldsymbol{\theta}_{\boldsymbol{K}}\right), \forall \vec{l}, \vec{j} \in \boldsymbol{S}$ are continuous and bounded functions from $\mathbb{R}^{d_{L}}$ to $\mathbb{R}^{+}$. 
- The physic equations $\overrightarrow{f_{L}}\left(\because, \mid \boldsymbol{\theta}_{L}\right), \forall \vec{\imath} \in \boldsymbol{S}$ are continuous functions from $\mathbb{R}^{d_{L}} \times \mathbb{R}^{+}$to $\mathbb{R}^{d_{L}}$ and locally Lipschitz continuous.

- The physic equations $\overrightarrow{f_{L}}\left(\cdot, t \mid \boldsymbol{\theta}_{L}\right), \forall \vec{\imath} \in \boldsymbol{S}$ are sub-linear, i.e. there are some $V_{1}>0$ and $V_{2}>0$ such that

$$
\forall \vec{x} \in \mathbb{R}^{d_{L}}, t \in \mathbb{R}^{+}\left|\overrightarrow{f_{L}^{\vec{\imath}}}\left(\vec{x}, t \mid \boldsymbol{\theta}_{L}\right)\right| \leq V_{1}(\|\vec{x}\|+|t|)+V_{2}
$$

- The functions $\operatorname{div}\left(\overrightarrow{f_{L}^{\vec{\imath}}}\left(\because, \mid \boldsymbol{\theta}_{L}\right)\right), \forall \vec{\imath} \in \boldsymbol{S}$ are almost everywhere bounded in absolute value by some real value $D>0$ (independent of $\vec{\imath}$ ).

\section{Solution approach}

Let $\overrightarrow{g^{\imath}}(\cdot, \cdot): \mathbb{R}^{d_{L}} \times \mathbb{R} \rightarrow \mathbb{R}^{d_{L}}$ denote the solution of

$$
\frac{\partial}{\partial t} \overrightarrow{g^{\imath}}\left(\vec{x}, t \mid \boldsymbol{\theta}_{L}\right)=\overrightarrow{f_{L}^{\vec{\imath}}}\left(\overrightarrow{g^{\imath}}\left(\vec{x}, t \mid \boldsymbol{\theta}_{L}\right), t \mid \boldsymbol{\theta}_{L}\right), \forall \vec{\imath} \in \boldsymbol{S}, \vec{x} \in \mathbb{R}^{d_{L}}, t \in \mathbb{R}
$$

with

$$
\overrightarrow{g^{\imath}}\left(\vec{x}, 0 \mid \boldsymbol{\theta}_{L}\right)=\vec{x}, \forall \vec{\imath} \in S, \overrightarrow{\mathrm{x}} \in \mathbb{R}^{\mathrm{d}_{\mathrm{L}}}
$$

and $\overrightarrow{g^{i}}\left(\vec{x}, t \mid \boldsymbol{\theta}_{L}\right)$ represents the deterministic evolution of $\vec{X}(t)$ at time $t$, starting from the condition $\vec{x}$ and while the processes $\vec{Y}(t)$ hold in state $\vec{\imath}$.

The state space $\mathbb{R}^{d_{L}}$ of continuous variables $\vec{X}(t)$ is divided into an admissible mesh $\mathcal{M}$, which is a family of measurable subsets of $\mathbb{R}^{d_{L}}\left(\mathcal{M}\right.$ is a partition of $\left.\mathbb{R}^{d_{L}}\right)$ such that:

(1) $\cup_{A \in \mathcal{M}} A=\mathbb{R}^{d_{L}}$.

(2) $\forall A, B \in \mathcal{M}, A \neq B \Rightarrow A \cap B=\emptyset$.

(3) $m_{A}=\int_{A} \overrightarrow{d x}>0, \forall A \in \mathcal{M}$, where $m_{A}$ is the volume of grid $A$.

(4) $\sup _{A \in \mathcal{M}} \operatorname{diam}(A)<+\infty$ where $\operatorname{diam}(A)=\sup _{\forall \vec{x}, \vec{y} \in A}|\vec{x}-\vec{y}|$.

Additionally, the time space $\mathbb{R}^{+}$is divided into small intervals $\mathbb{R}^{+}=\bigcup_{n=0,1,2, \ldots}[n \Delta t,(n+1) \Delta t[$, by setting the time step $\Delta t>0$ (the length of each interval).

Let $p_{t}\left(d \vec{z} \mid \boldsymbol{\theta}=\boldsymbol{\theta}_{L} \cup \boldsymbol{\theta}_{K}\right)$ denote the probability distribution of $\vec{Z}(t)$. The numerical scheme aims at constructing an approximate value $\rho_{t}(\vec{x}, \mid \boldsymbol{\theta}) d \vec{x}$ for $p_{t}(d \vec{x} ; \mid \boldsymbol{\theta})$, such that $\rho_{t}(\vec{x} ; \mid \boldsymbol{\theta})$ is constant on each $\left[n \Delta t,(n+1) \Delta t\left[\times A \times\left\{\boldsymbol{y}_{i}\right\}\right.\right.$, $\forall A \in \mathcal{M}, \vec{i} \in \boldsymbol{S}$ :

$$
\rho_{t}(\vec{x}, \vec{\imath} \mid \boldsymbol{\theta})=P_{n}(A, \vec{\imath} \mid \boldsymbol{\theta}), \forall \vec{\imath} \in \boldsymbol{S}, \vec{x} \in A, t \in[n \Delta t,(n+1) \Delta t[
$$

$P_{0}(A, \vec{\imath} \mid \boldsymbol{\theta}), \forall \vec{\imath} \in \boldsymbol{S}, A \in \mathcal{M}$ is defined as follows: 


$$
P_{0}(A, \vec{\imath} \mid \boldsymbol{\theta})=\int_{A} p_{0}(d \vec{x}, \vec{\imath} \mid \boldsymbol{\theta}) / m_{A}
$$

Then, $\quad P_{n+1}(A, \vec{\imath} \mid \boldsymbol{\theta}), \forall \vec{\imath} \in \boldsymbol{S}, A \in \mathcal{M}, n \in \mathbb{N}$ can be calculated considering the deterministic evaluation of $\vec{X}(t)$ and the stochastic evolution of $\vec{Y}(t)$ based on $P_{n}(\mathcal{M}, \vec{\imath} \mid \boldsymbol{\theta})$ by the Chapman-Kolmogorov forward equation, as follows:

$$
\begin{gathered}
P_{n+1}(A, \vec{\imath} \mid \boldsymbol{\theta}) \\
=\frac{1}{1+\Delta t b_{A}^{\vec{\imath}}} \widehat{P_{n+1}}(A, \vec{\imath} \mid \boldsymbol{\theta})+\Delta t \sum_{\vec{\jmath} \in S} \frac{a_{A}^{\vec{j} \vec{\imath}}}{1+\Delta t b_{A}^{\vec{j}}} \widehat{P_{n+1}}(A, \vec{\jmath} \mid \boldsymbol{\theta})
\end{gathered}
$$

where

$$
a_{A}^{\vec{\jmath} \vec{l}}=\int_{A} \lambda_{\vec{j}}\left(\vec{l}, \vec{x} \mid \boldsymbol{\theta}_{K}\right) \overrightarrow{d x} / m_{A}, \forall \vec{\imath} \in \boldsymbol{S}, A \in \mathcal{M}
$$

is the average transition rate from state $\vec{\jmath}$ to state $\vec{\imath}$ for grid $A$,

$$
b_{A}^{\vec{\imath}}=\sum_{\vec{\jmath} \neq \vec{\imath}} a_{A}^{\vec{\imath} \vec{\jmath}}, \forall \vec{\imath} \in S, A \in \mathcal{M}
$$

is the average transition rate out of state $\vec{\imath}$ for grid $A$,

$$
\widehat{P_{n+1}}(A, \vec{\imath} \mid \boldsymbol{\theta})=\sum_{B \in \mathcal{M}} m_{B A}^{\vec{\imath}} P_{n}(B, \vec{\imath} \mid \boldsymbol{\theta}) / m_{A}, \forall \vec{\imath} \in \boldsymbol{S}, A \in \mathcal{M}
$$

is the approximate value of probability density function on $\{\vec{l}\} \times[(n+1) \Delta t,(n+$ 2) $\Delta t[\times A$ according to the deterministic evolution of $\vec{X}(t)$,

$$
m_{B A}^{\vec{\imath}}=\int_{\left\{\vec{y} \in B \mid \overrightarrow{g^{\imath}}\left(\vec{y}, \Delta t \mid \theta_{L}\right) \in A\right\}} \overrightarrow{d y}, \forall \vec{\imath} \in S, A, B \in \mathcal{M}
$$

is the volume of the part of grid $B$ which will enter grid $A$ after time $\Delta t$, according to the deterministic evolution of $\vec{X}(t)$.

Fig. 14 shows an illustrative example in $\mathbb{R}^{2}$ to explain the procedure of FV scheme.

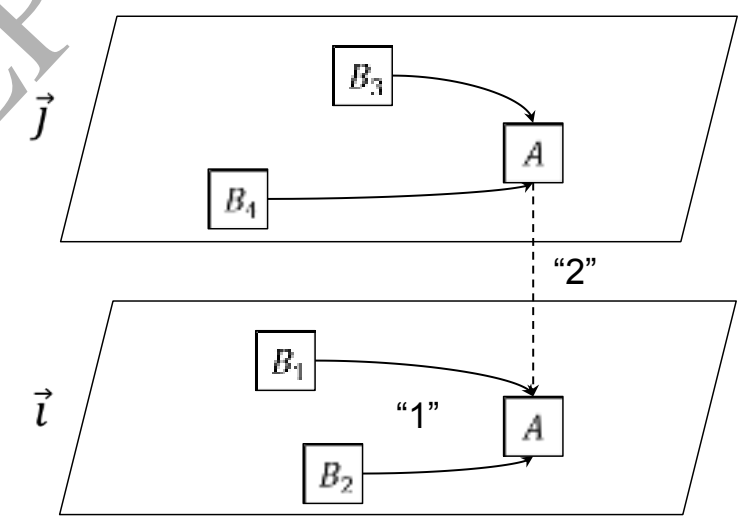

Fig. 14. The evolution of degradation processes during $[n \Delta t,(n+1) \Delta t]$. [31]

FV scheme solves PDMP by considering two different situations to calculate the 
probability that $\vec{Z}(t) \in(\mathrm{A}, \vec{\imath}), \forall \vec{\imath} \in S, A \in \mathcal{M}$ at time $(n+1) \Delta t$, according to eq. (34). The first one (denoted by "1" in Fig. 14) is that $\vec{X}(t)$ evolves but $\vec{Y}(t)$ doesn't change, which is quantified by the first term of right-hand parts of eq. (34) where $\frac{1}{1+\Delta t b_{A}^{\imath}}$ is the approximated probability that no transition happens from state $\vec{\imath}$ for grid $A\left(B_{1}, B_{2}\right.$ are the grids of which some parts will enter grid $A$ according to the deterministic evaluation of $\vec{X}(t)$ at time $(n+1) \Delta t$ given $\vec{Y}(t)=\vec{\imath})$. The second one (denoted by " 2 " in Fig. 14) is that $\vec{X}(t)$ evolves and $\vec{Y}(t)$ step to state $\vec{\imath}$ from another state $\vec{\jmath} \in S$, which is quantified by the second term of right-hand parts of eq. (34), where $a_{A}^{\vec{\jmath} \vec{l}} \Delta t$ is the transition probability from state $\vec{\jmath}$ to state $\vec{\imath}$ for grid $A$ $\left(B_{3}, B_{4}\right.$ are the grids of which some parts will enter grid $A$ according to the deterministic evaluation of $\vec{X}(t)$ at time $(n+1) \Delta t$ given $\vec{Y}(t)=\vec{\jmath}$.

The approximated solution $\rho_{t}(\vec{x} ; \mid \boldsymbol{\theta}) d \vec{x}$ weakly converges towards $p_{t}(d \vec{x} ; \mid \boldsymbol{\theta})$ when $\Delta t \rightarrow 0$ and $|\mathcal{M}| / \Delta t \rightarrow 0$ where $|\mathcal{M}|=\sup _{A \in \mathcal{M}} \operatorname{diam}(A)$. The reliability of the system can, then, be calculated as follows:

$$
R(t)=\int_{\vec{z} \notin \mathcal{F}} p_{t}(d \vec{z} \mid \boldsymbol{\theta})
$$

